\title{
Modeling of Non-WSSUS Double-Rayleigh Fading Channels for Vehicular Communications
}

\author{
Carlos A. Gutiérrez, ${ }^{1}$ J. J. Jaime-Rodríguez, ${ }^{1}$ J. M. Luna-Rivera, ${ }^{1}$ \\ Daniel U. Campos-Delgado, ${ }^{1}$ and Javier Vázquez Castillo ${ }^{2}$ \\ ${ }^{1}$ Faculty of Science, Universidad Autónoma de San Luis Potosí, Av. Salvador Nava Martinez s/n, 78290 San Luis Potosí, SLP, Mexico \\ ${ }^{2}$ Department of Engineering, Universidad de Quintana Roo, Blvd. Bahía Esq. Ignacio Comonfort s/n, 77019 Chetumal, QR, Mexico
}

Correspondence should be addressed to Carlos A. Gutiérrez; cagutierrez@fc.uaslp.mx

Received 29 April 2017; Revised 14 July 2017; Accepted 1 August 2017; Published 3 October 2017

Academic Editor: Xianfu Lei

Copyright (C) 2017 Carlos A. Gutiérrez et al. This is an open access article distributed under the Creative Commons Attribution License, which permits unrestricted use, distribution, and reproduction in any medium, provided the original work is properly cited.

\begin{abstract}
This paper deals with the modeling of nonstationary time-frequency (TF) dispersive multipath fading channels for vehicle-tovehicle $(\mathrm{V} 2 \mathrm{~V})$ communication systems. As a main contribution, the paper presents a novel geometry-based statistical channel model that facilitates the analysis of the nonstationarities of $\mathrm{V} 2 \mathrm{~V}$ fading channels arising at a small-scale level due to the timevarying nature of the propagation delays. This new geometrical channel model has been formulated following the principles of plane wave propagation (PWP) and assuming that the transmitted signal reaches the receiver antenna through double interactions with multiple interfering objects (IOs) randomly located in the propagation area. As a consequence of such interactions, the firstorder statistics of the channel model's envelope are shown to follow a worse-than-Rayleigh distribution; specifically, they follow a double-Rayleigh distribution. General expressions are derived for the envelope and phase distributions, four-dimensional (4D) TF correlation function (TF-CF), and TF-dependent delay and Doppler profiles of the proposed channel model. Such expressions are valid regardless of the underlying geometry of the propagation area. Furthermore, a closed-form solution of the 4D TF-CF is presented for the particular case of the geometrical two-ring scattering model. The obtained results provide new theoretical insights into the correlation and spectral properties of small-scale nonstationary V2V double-Rayleigh fading channels.
\end{abstract}

\section{Introduction}

Terrestrial vehicle-to-vehicle (V2V) communication systems are emerging as an enabling technology for a variety of new wireless applications and services, such as information relaying for mobile cellular networks [1] and peer-to-peer data transmission for vehicular communications [2]. Some of the most important applications of these systems target the prevention of vehicular accidents and the optimization of traffic flow. Such applications have caught the attention of the automotive industry and different government bodies around the world, who have become major promoters of the $\mathrm{V} 2 \mathrm{~V}$ communications technology [3].

One of the main challenges in the design of $\mathrm{V} 2 \mathrm{~V}$ communication systems is to develop a robust air interface that supports delay sensitive applications under the constraints of a rapidly changing propagation environment and a dynamic network topology. To successfully design and optimize such an air interface, a realistic reference model of the timefrequency (TF) dispersive V2V fading channel is required. This is of primary importance, since the performance of the wireless communication systems is highly influenced by the propagation environment. In addition to measurementbased models, proper analytic channel models are needed that provide insights into the physics of $\mathrm{V} 2 \mathrm{~V}$ radio reception and, at the same time, that lend themselves to mathematical and numerical system performance investigations.

Important advances in the analytical characterization of fixed-to-mobile (F2M) multipath fading channels were prompted by the emergence of the mobile cellular communication systems in the late 1970s. However, the modeling of fading channels for V2V communications not only demands more exhaustive research work, but also requires a shift of paradigm, because some of the assumptions that are often 
invoked for the characterization of F2M channels are not valid when the terminals at both ends of the radio link are able to move at high speeds. For example, most of the existing statistical models for F2M channels have been formulated assuming the fulfillment of the wide-sense stationary uncorrelated scattering (WSSUS) condition introduced by Bello in [4] (e.g., see [5-7]). This assumption facilitates the mathematical analysis of TF-dispersive channels, as it implies that the channel's statistics are simultaneously wide-sense stationary (WSS) in the time and the frequency domains. Nevertheless, recent empirical investigations carried out in vehicular communication environments suggest that the WSSUS condition is not valid for V2V channels [8]. While the nonstationary features of multipath wireless channels have been a subject of analysis since the early days of the mobile radio communications (e.g., see $[4,9]$ ), the modeling of such nonstationarities has predominantly been addressed from a large-scale propagation perspective. Measured data obtained independently in [10-12] demonstrates that the nonstationary characteristics of $\mathrm{V} 2 \mathrm{~V}$ channels are also meaningful at a small-scale level.

Empirical investigations have further shown that the signal fading induced by V2V channels is more severe than the one produced by F2M channels [13], which is typically modeled by Rayleigh or Rice distributions. The exacerbation of signal fading is not surprising if one considers that, in a V2V communications system, the mobile terminals are located at ground level. This scenario increases the chances of receiving echoes of the transmitted signal that interact with multiple interfering objects (IOs) on their way to the receiver antenna. As a result of such multiple interactions, the received multipath signal is subject to a form of cascaded fading that is modeled by worse-than-Rayleigh distributions [13, 14] (e.g., the double-Rayleigh [15] and double-Rice distributions [16]).

Notable contributions to the analytical characterization of non-WSSUS V2V channels have recently been made in [1719] on the basis of the geometry-based statistical modeling approach. The geometrical channel models proposed in these papers assume the propagation of spherical waves to account for the nonstationarities of $\mathrm{V} 2 \mathrm{~V}$ channels stemming from small-scale propagation. In the spherical wave propagation (SWP) framework, the angle of departure (AOD) and angle of arrival (AOA) of the received multipath signals are determined by the instantaneous spatial position of the transmitting and receiving mobile stations (MSs). The angular statistics of the resulting channel models are therefore timedependent. This feature is particularly convenient for the characterization of nonstationary small-scale channels but renders the mathematical analysis of the channel's statistics a cumbersome task.

To facilitate the modeling and analysis of non-WSSUS $\mathrm{V} 2 \mathrm{~V}$ channels, we recently proposed in [20-22] a novel framework that builds instead on the principles of plane wave propagation (PWP). Our proposal is well suited for the analysis of $\mathrm{V} 2 \mathrm{~V}$ radio reception over small local areas spanning a few tens of wavelengths, where a plane wave approximation of the more realistic spherical electromagnetic waves can be applied. For such propagation scenarios, the angular statistics of the $\mathrm{V} 2 \mathrm{~V}$ channel can be modeled by time-invariant distributions, which are more mathematically tractable than their time-varying counterparts. The focus of $[20,21]$ was on the characterization of nonstationary Rayleigh fading channels for single-input single-output (SISO) and multiple-input multiple-output (MIMO) V2V communication systems, respectively. In [21], the MSs are assumed to move at constant speeds and on linear trajectories, whereas the effects of acceleration and nonlinear motion are investigated in [20] following a parallel approach to the TF analysis techniques employed in [23]. On the other hand, in [22], we apply our modeling framework to the characterization of non-WSSUS SISO V2V channels that experience doubleRayleigh fading.

In this paper, we complete our preliminary work presented in [22] by providing a detailed description and a thorough statistical analysis of the proposed geometrical model for non-WSSUS V2V double-Rayleigh fading channels. The scope and depth of the work in [22] are expanded here as follows:

(i) Important statistical quantities of the proposed channel model, such as the autocorrelation functions in the time and frequency domains, as well as the TF-dependent delay and Doppler profiles, were not investigated in [22]. An in-depth analysis of these statistical quantities is presented here.

(ii) Details on the derivations of the four-dimensional (4D) TF correlation function (4D TF-CF) of the proposed channel model were not presented in [22]. An outline of the derivations is given here in the Appendix.

(iii) In [22], our discussion of the proposed channel model's stationary (nonstationary) characteristics was constrained to a single paragraph due to space limitations. In this paper, we complement our discussion with important additional remarks.

(iv) Finally, new numerical examples are presented in this paper to illustrate our findings regarding the autocorrelation, spectral, and stationary (nonstationary) characteristics of the proposed geometry-based statistical model (GBSM) for non-WSSUS V2V doubleRayleigh fading channels.

The remainder of the paper is organized as follows. Our proposal for the geometrical modeling of nonstationary V2V double-Rayleigh fading channels is presented in Section 2. In Section 3, we derive general expressions for the envelope and phase distributions, the 4D TF-CF, and the TF-dependent delay and Doppler profiles of the proposed channel model. It is worth pointing out that such expressions are valid regardless of the underlying geometry of the propagation area. In Section 4, we compute a closed-form solution of the $4 \mathrm{D}$ TF-CF by considering the particular case of the geometrical two-ring scattering model. Numerical examples illustrating our theoretical findings are presented in Section 4. Finally, our conclusions are given in Section 5.

Notation. The complex conjugate, the argument, and the absolute value operations are denoted by $(\cdot)^{*}, \arg \{\cdot\}$, and $|\cdot|$, 


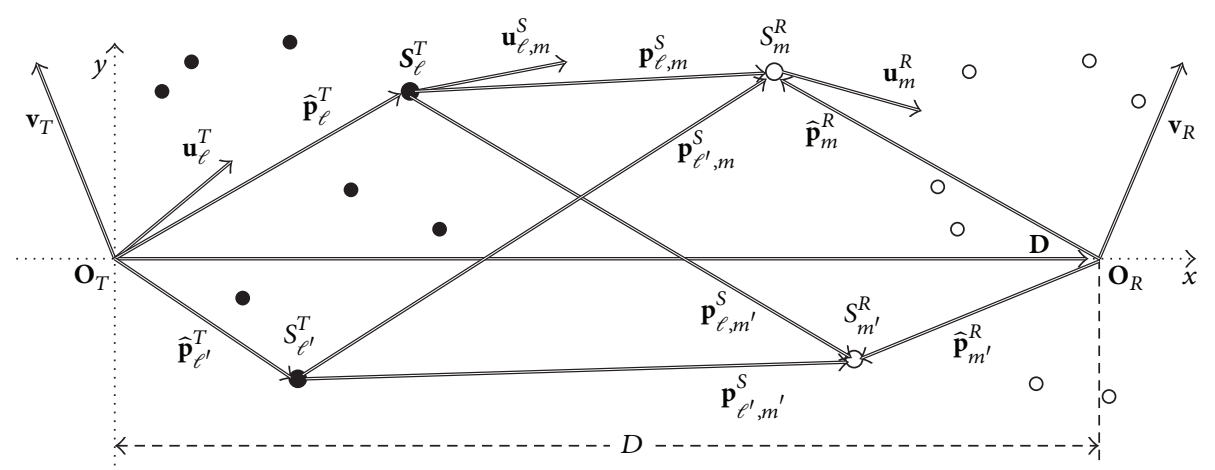

FIGURE 1: The reference propagation scenario at time $t=t_{0}$.

respectively. Vectors are written in bold face. The transpose operation is denoted by $(\cdot)^{\dagger},\|\cdot\|$ stands for the Euclidean norm, and the scalar product between two vectors $\mathbf{z}_{1}$ and $\mathbf{z}_{2}$ is represented as $\left\langle\mathbf{z}_{1}, \mathbf{z}_{2}\right\rangle$. The operator $E\{\cdot\}$ designates the statistical expectation. The set of positive real numbers is denoted by $\mathbb{R}^{+}$, and the operator $\operatorname{card}(\cdot)$ indicates set cardinality.

\section{The Proposed Geometrical Model for Non-WSSUS V2V Channels}

2.1. Geometrical Modeling of the Propagation Scenario. The aim of this paper is to model the nonstationarities of TFdispersive $\mathrm{V} 2 \mathrm{~V}$ channels stemming from small-scale propagation. We are particularly interested in characterizing the nonstationarities arising from the time-varying nature of the propagation delays. For that purpose, we consider a SISO V2V communication system and assume that the transmitted signal reaches the receiver antenna through a double interaction with nonmoving IOs randomly located in the propagation environment. Specifically, we assume that the transmitted signal interacts first with a set $\mathbb{S}_{T}$ of $L$ IOs $\left(\operatorname{card}\left(\mathbb{S}_{T}\right)=L\right)$ that are located in the surroundings of the transmitting $\mathrm{MS}\left(T_{X}\right)$. Then, the $L$ scattered signals that result from such an interaction impinge on a second set $\mathbb{S}_{R}$ of $M$ IOs $\left(\operatorname{card}\left(\mathbb{S}_{R}\right)=M\right)$ that lie in the vicinity of the receiving MS $\left(R_{X}\right)$. Thereby, a total of $L \times M$ double-scattered waves are produced, which combine with one another at the receiver antenna. Figure 1 shows an illustration of the propagation scenario under consideration at the time instant $t=t_{0}$ when the MSs start to communicate with each other.

In Figure 1, the $L$ IOs in the set $\mathbb{S}_{T}$ are represented by black dots, whereas white dots stand for the $M$ IOs in $\mathbb{S}_{R}$. We denote the $l$ th IO in $\mathbb{S}_{k}$ by $S_{l}^{k}$, for $l \in\left\{1,2, \ldots, \operatorname{card}\left(\mathbb{S}_{k}\right)\right\}$, and $k \in\{T, R\}$. The positions of $T_{X}$ and $R_{X}$ at the time when the communications begin (i.e., at $t=t_{0}$ ) are denoted by the time-invariant vectors $\mathbf{O}_{T}$ and $\mathbf{O}_{R}$. The distance between $\mathbf{O}_{T}$ and $\mathbf{O}_{R}$ is given by $D$. The velocity vectors of $T_{X}$ and $R_{X}$ are represented by $\mathbf{v}_{T}$ and $\mathbf{v}_{R}$, respectively. We assume that $T_{X}$ and $R_{X}$ move at constant speeds and on linear trajectories.

The time-invariant vectors $\widehat{\mathbf{p}}_{l}^{k}$, for $l \in\left\{1,2, \ldots, \operatorname{card}\left(\mathbb{S}_{k}\right)\right\}$ and $k \in\{T, R\}$, indicate the position of the $l$ th IO $S_{l}^{k}$ in $\mathbb{S}_{k}$, with respect to the fixed reference point $\mathbf{O}_{k}$. In addition, the instantaneous position of the $\ell$ th $\mathrm{IO} S_{\ell}^{T}$ in $\mathbb{S}_{T}$, as seen from the perspective of the moving $T_{X}$, can be represented by the time-varying vector $\mathbf{p}_{\ell}^{T}(t)$. Analogously, the time-varying vector $\mathbf{p}_{m}^{R}(t)$ indicates the instantaneous position of the $m$ th fixed IO $S_{m}^{R}$ in $\mathbb{S}_{R}$, as seen by the moving $R_{X}$. Regardless of the geometrical configuration of the propagation scenario, we can express these two vectors as

$$
\mathbf{p}_{l}^{k}(t)=\widehat{\mathbf{p}}_{l}^{k}-t \cdot \mathbf{v}_{k}
$$

for $k \in\{T, R\}$ and $l \in\left\{1,2, \ldots, \operatorname{card}\left(\mathbb{S}_{k}\right)\right\}$. On the other hand, the position of the $m$ th nonmoving $\operatorname{IO} S_{m}^{R}$ in $\mathbb{S}_{R}$ relative to that of the $\ell$ th IO $S_{\ell}^{T}$ in $\mathbb{S}_{T}$ is given by the time-invariant vector

$$
\mathbf{p}_{\ell, m}^{S}=\mathbf{D}-\widehat{\mathbf{p}}_{\ell}^{T}+\widehat{\mathbf{p}}_{m}^{R}
$$

for $\ell \in\left\{1,2, \ldots, \operatorname{card}\left(\mathbb{S}_{T}\right)\right\}$ and $m \in\left\{1,2, \ldots, \operatorname{card}\left(\mathbb{S}_{R}\right)\right\}$, where $\mathbf{D}=\mathbf{O}_{R}-\mathbf{O}_{T}$ and $\|\mathbf{D}\|=D$. The time-invariant vectors $\mathbf{u}_{\ell}^{T}, \mathbf{u}_{\ell, m}^{S}$, and $\mathbf{u}_{m}^{R}$ are unit vectors which point at the direction of propagation of the waves that travel from $T_{X}$ to $S_{\ell}^{T}$, from $S_{\ell}^{T}$ to $S_{m}^{R}$, and from $S_{m}^{R}$ to $R_{X}$, respectively. Note that these vectors do not need to point exactly towards $S_{\ell}^{T}, S_{m}^{R}$, and $\mathbf{O}_{R}$, if we assume (as we do in this paper) the propagation of plane waves. If, on the other hand, we consider the propagation of spherical waves, then such vectors should point exactly towards the aforementioned observation points. In fact, in the case of SWP, $\mathbf{u}_{\ell}^{T}$ and $\mathbf{u}_{m}^{R}$ should be modeled as timevarying vectors, since the position of the observer relative to the source changes over time because $T_{X}$ and $R_{X}$ are moving [18].

Geometrical configurations of the propagation scenario similar to the one shown in Figure 1 have already been considered in other papers for modeling SISO and MIMO V2V fading channels (e.g., see [24-26]). However, the relative positions among the MSs and the IOs are characterized in this paper following a vector framework that allows capturing the temporal dynamics of the channel in a simple and compact manner. In contrast, such relative positions are typically modeled in the state of the art by a framework of time-invariant scalar quantities (angles and distances) that provide a less flexible description of the propagation scenario's temporal dynamics. Capitalizing on the flexibility of the vector framework presented here, we propose in the 
following subsection a general model for the channel impulse response (CIR) of V2V fading channels that is valid regardless of the geometrical configuration of the propagation area, as long as the plane wave model applies and the transmitted signal arrives at $R_{X}$ via a double interaction with IOs.

\subsection{Mathematical Model of the Channel Impulse Response.} We define the small-scale CIR in the equivalent baseband at time $t$ due to an impulse applied $\tau$ seconds in the past by the superposition of $L \times M$ plane waves as follows:

$$
\begin{aligned}
h(t ; \tau) \triangleq & \Pi_{T_{0}}\left(t-t_{0}\right) \sum_{\ell=1}^{L} \sum_{m=1}^{M} g_{\ell}^{T} g_{m}^{R} \\
& \cdot \exp \left\{j\left[\theta_{0}-\theta_{\ell}^{T}-\theta_{m}^{R}-\vartheta_{\ell, m}(t)\right]\right\} \\
& \cdot \delta\left(\tau-\tau_{\ell, m}(t)\right) .
\end{aligned}
$$

In (3), $j^{2}=-1 ; \theta_{0}$ is the initial phase of the transmitted signal; $g_{l}^{k}$ and $\theta_{l}^{k}$ stand for the gain and phase shift, respectively, introduced by the interaction of the transmitted signal with the $l$ th IO $S_{l}^{k}$ in $\mathbb{S}_{k}$, for $l \in\left\{1,2, \ldots, \operatorname{card}\left(\mathbb{S}_{k}\right)\right\}$ and $k \in\{T, R\}$. The Dirac delta function is denoted by $\delta(\cdot)$, and

$$
\Pi_{T_{0}}\left(t-t_{0}\right) \triangleq \begin{cases}1, & t_{0} \leq t \leq t_{0}+T_{0} \\ 0, & \text { otherwise }\end{cases}
$$

is a rectangular windowing function introduced as a means to limit the length of the CIR $h(t ; \tau)$ within an interval of length $T_{0}$ inside of which the large-scale variations of the channel are negligible, and the plane wave approximation is reasonably justified. For simplicity, and without loss of generality, we will henceforth assume that $t_{0}=0$.

The time-varying parameters $\tau_{\ell, m}(t)$ and $\vartheta_{\ell, m}(t)$ stand for the instantaneous propagation delay and path-length dependent phase rotation, respectively, of the multipath signal that arrives at $R_{X}$ via the interaction with the IOs $S_{\ell}^{T}$ and $S_{m}^{R}$. These two parameters are related to each other by

$$
\vartheta_{\ell, m}(t)=2 \pi f_{c} \tau_{\ell, m}(t)
$$

where $f_{c}=C_{l} / \lambda$ is the carrier frequency, $C_{l}$ denotes the speed of light, and $\lambda$ is the transmitted signal's wavelength. We model the propagation delays as

$$
\tau_{\ell, m}(t)=\frac{\left\langle\mathbf{p}_{\ell}^{T}(t), \mathbf{u}_{\ell}^{T}\right\rangle+\left\langle\mathbf{p}_{\ell, m}^{S}, \mathbf{u}_{\ell, m}^{S}\right\rangle-\left\langle\mathbf{p}_{m}^{R}(t), \mathbf{u}_{m}^{R}\right\rangle}{C_{l}}
$$

The sum of scalar products at the right-hand side of (6) provides the path length of the plane wave that travels from $T_{X}$ to $R_{X}$ via $S_{\ell}^{T}$ and $S_{m}^{R}$ [27]. The path length is computed in (6) by considering the instantaneous position of the moving $T_{X}$ and $R_{X}$, which is accounted for by the time-varying position vectors $\mathbf{p}_{\ell}^{T}(t)$ and $\mathbf{p}_{m}^{R}(t)$. Equation (6) therefore allows modeling the time-varying nature of the propagation delays. This is a salient feature of our proposal, because to the best of our knowledge, the GBSMs for V2V channels that have been proposed under similar considerations (i.e., by assuming a double interaction with IOs and the propagation of plane waves) do not take into account the temporal dynamics of the propagation delays (see, e.g., [24-26]). Such geometrical channel models define the propagation delays as time-invariant quantities $\tau_{\ell, m}$ that depend only on the position of $T_{X}$ and $R_{X}$ at time $t=0$. (When the propagation of plane waves is assumed, the propagation delays are modeled in the state of the art as $\tau_{\ell, m}=\left[\left\langle\widehat{\mathbf{p}}_{\ell}^{T}, \mathbf{u}_{\ell}^{T}\right\rangle+\left\langle\mathbf{p}_{\ell, m}^{S}, \mathbf{u}_{\ell, m}^{S}\right\rangle-\right.$ $\left.\left.\left\langle\widehat{\mathbf{p}}_{\ell}^{R}, \mathbf{u}_{m}^{R}\right\rangle\right] / C_{l}, \forall m.\right)$

From (1), and assuming that the unit vectors $\mathbf{u}_{\ell}^{T}, \mathbf{u}_{\ell, m}^{S}$, and $\mathbf{u}_{m}^{R}$ are collinear with the time-invariant vectors $\widehat{\mathbf{p}}_{\ell}^{T}, \mathbf{p}_{\ell, m}^{S}$, and $\widehat{\mathbf{p}}_{m}^{R}$, respectively, in such a way that

$$
\begin{aligned}
\left\langle\widehat{\mathbf{p}}_{\ell}^{T}, \mathbf{u}_{\ell}^{T}\right\rangle & =\left\|\widehat{\mathbf{p}}_{\ell}^{T}\right\| \\
\left\langle\mathbf{p}_{\ell, m}^{S}, \mathbf{u}_{\ell, m}^{S}\right\rangle & =\left\|\mathbf{p}_{\ell, m}^{S}\right\| \\
\left\langle\widehat{\mathbf{p}}_{m}^{R}, \mathbf{u}_{m}^{R}\right\rangle & =-\left\|\widehat{\mathbf{p}}_{m}^{R}\right\|,
\end{aligned}
$$

we have

$$
\begin{aligned}
\left\langle\mathbf{p}_{\ell}^{T}(t), \mathbf{u}_{\ell}^{T}\right\rangle & =\left\|\widehat{\mathbf{p}}_{\ell}^{T}\right\|-t\left\langle\mathbf{v}_{T}, \mathbf{u}_{\ell}^{T}\right\rangle \\
\left\langle\mathbf{p}_{\ell, m}^{S}, \mathbf{u}_{\ell, m}^{S}\right\rangle & =\left\|\mathbf{p}_{\ell, m}^{S}\right\|=\left\|\mathbf{D}-\widehat{\mathbf{p}}_{\ell}^{T}+\widehat{\mathbf{p}}_{m}^{R}\right\| \\
\left\langle\mathbf{p}_{m}^{R}(t), \mathbf{u}_{m}^{R}\right\rangle & =-\left\|\widehat{\mathbf{p}}_{m}^{R}\right\|-t\left\langle\mathbf{v}_{R}, \mathbf{u}_{m}^{R}\right\rangle .
\end{aligned}
$$

Thereby, the propagation delays $\tau_{\ell, m}(t)$ in (6) can be written as

$$
\tau_{\ell, m}(t)=\frac{\left\|\widehat{\mathbf{p}}_{\ell}^{T}\right\|+\left\|\mathbf{p}_{\ell, m}^{S}\right\|+\left\|\widehat{\mathbf{p}}_{m}^{R}\right\|}{C_{l}}-t \frac{f_{\ell, m}^{D}}{f_{c}}
$$

where

$$
f_{\ell, m}^{D}=f_{\ell}^{T}+f_{m}^{R}
$$

is a Doppler frequency shift due to the combined movement of $T_{X}$ and $R_{X}$, and

$$
\begin{gathered}
f_{\ell}^{T}=\frac{\left\langle\mathbf{v}_{T}, \mathbf{u}_{\ell}^{T}\right\rangle}{\lambda} \\
f_{m}^{R}=-\frac{\left\langle\mathbf{v}_{R}, \mathbf{u}_{m}^{R}\right\rangle}{\lambda} .
\end{gathered}
$$

It is worth highlighting that the channel modeling framework defined by (3)-(16) is not restricted to a particular geometrical arrangement of the IOs' positions. Moreover, the proposed framework is valid for both two-dimensional (2D) and three-dimensional (3D) propagation scenarios, as it applies regardless of whether the vectors introduced in Figure 1 are defined in 2D or 3D spaces. 


\section{Statistical Properties of the Proposed Non-WSSUS V2V Channel Model}

3.1. Considerations. In this section, we analyze the first-order (FO) statistics, the correlation properties, and the spectral characteristics of the proposed GBSM for V2V channels. To reduce the number of variables in our analysis, we will restrict our attention to a $2 \mathrm{D}$ propagation scenario. We point out, however, that the extension with respect to $3 \mathrm{D}$ propagation is straightforward. In addition, we will make the following considerations:

(i) The gains $g_{l}^{k}$ in (3) are statistically independent, although not necessarily identically distributed, positive random variables (r.v.), each having a probability density function (PDF) $p_{g_{l}}(c)$, for $l \in$ $\left\{1,2, \ldots, \operatorname{card}\left(\mathbb{S}_{k}\right)\right\}, k \in\{T, R\}$, and $c \geq 0$.

(ii) The phases $\theta_{l}^{k}, l \in\left\{1,2, \ldots, \operatorname{card}\left(\mathbb{S}_{k}\right)\right\}, k \in\{T, R\}$, are statistically independent r.v. uniformly distributed over $[-\pi, \pi)$.

(iii) The direction of the time-invariant position vector $\widehat{\mathbf{p}}_{\ell}^{T}$ is modeled by a random angle $\phi_{\ell}^{T}$ characterized by a circular $\operatorname{PDF} p_{\phi}^{T}\left(\phi_{T}\right)$, for all $\ell$, and $\phi_{T} \in[-\pi, \pi)$. Due to the collinearity between $\widehat{\mathbf{p}}_{\ell}^{T}$ and $\mathbf{u}_{\ell}$ (see (7)), $\phi_{\ell}^{T}$ can be identified as the AOD of the plane wave that interacts with the $\ell$ th IO $S_{\ell}^{T}$ in $\mathbb{S}_{T}$.

(iv) The r.v. $\phi_{m}^{R}$ describes the direction of the timeinvariant position vector $\widehat{\mathbf{p}}_{m}^{R}$ and is characterized by a circular PDF $p_{\phi}^{R}\left(\phi_{R}\right)$, for all $\ell$, and $\phi_{R} \in[-\pi, \pi)$. Due to the collinearity between $\widehat{\mathbf{p}}_{m}^{R}$ and $\mathbf{u}_{m}$ (see (9)), we can refer to $\phi_{m}^{R}$ as the AOA of the $m$ th multipath signal that arrives at $R_{X}$ through the interaction with the $m$ th IO $S_{m}^{R}$ in $\mathbb{S}_{R}$.

(v) The time-invariant vectors $\widehat{\mathbf{p}}_{\ell}^{T}$ and $\widehat{\mathbf{p}}_{m}^{R}$ have magnitude modeled by functions of $\phi_{\ell}^{T}$ and $\phi_{m}^{R}$, respectively; that is,

$$
\left\|\widehat{\mathbf{p}}_{l}^{k}\right\|=G_{k}\left(\phi_{l}\right), \quad G_{k}:[-\pi, \pi) \longmapsto \mathbb{R}^{+}
$$

for $l \in\left\{1,2, \ldots, \operatorname{card}\left\{\mathbb{S}_{k}\right\}\right\}$ and $k \in\{T, R\}$.

(vi) The aforementioned gains, phases, AODs, and AOAs are mutually independent r.v.

Assumptions (i)-(iv) and (vi) are customary in the state of the art, but the one introduced in (v) is not. This latter assumption provides a generic description of the IOs' position and is introduced in this paper with the purpose of obtaining insights into the channel model's statistics that are not limited to a specific geometrical configuration of the propagation area. The fact that the magnitude of $\widehat{\mathbf{p}}_{\ell}^{T}$ and $\widehat{\mathbf{p}}_{m}^{R}$ is modeled as functions of $\phi_{\ell}^{T}$ and $\phi_{m}^{R}$ implies that the IOs are located on the contour of two surfaces. The shape of such surfaces will be determined by the functions $G_{T}$ and $G_{R}$.
3.2. First-Order Statistical Analysis. The instantaneous mean value and average power of a doubly dispersive V2V fading channel are TF-varying quantities given as $m_{h}(t ; f) \triangleq$ $E\{H(t ; f)\}$ and $P_{h}(t ; f) \triangleq E\left\{|H(t ; f)|^{2}\right\}$, where $H(t ; f) \triangleq$ $\int_{-\infty}^{\infty} h(t ; \tau) \exp \{-j 2 \pi f \tau\} d \tau$ is the channel transfer function. By computing the Fourier transform of the CIR in (3) with respect to $\tau$, we find that

$$
\begin{aligned}
H(t ; f)= & \Pi_{T_{0}}(t) \sum_{\ell=1}^{L} \sum_{m=1}^{M} g_{\ell}^{T} g_{m}^{R} \exp \left\{j\left[\theta_{0}-\theta_{\ell}^{T}-\theta_{m}^{R}\right]\right\} \\
& \cdot \exp \left\{-j 2 \pi\left(f_{c}+f\right) \tau_{\ell, m}(t)\right\} .
\end{aligned}
$$

Under Assumptions (i)-(vi), we find $m_{h}(t ; f)=0$ and $P_{h}(t ; f)=\sigma_{H}=\zeta_{T} \zeta_{R}$, for $t \in\left[0, T_{0}\right]$, where

$$
\zeta_{k}=\sum_{l=1}^{\operatorname{card}\left\{\mathbb{S}_{k}\right\}} E\left\{\left|g_{l}^{k}\right|^{2}\right\}>0, \quad \text { for } k \in\{T, R\} .
$$

The previous equation indicates that the average power of the channel is just a cumulus of the average powers of the received multipath signal's components (as was to be expected). This equation also shows that the channel's largescale variations are not taken into account by the model in (18), as the instantaneous average power of $H(t ; f)$ is a TFinvariant quantity for $t \in\left[0, T_{0}\right]$. We have intentionally neglected such variations to focus the spotlight of our work on the nonstationarities of $\mathrm{V} 2 \mathrm{~V}$ channels arising from the time-varying nature of the propagation delays. However, the large-scale variations can easily be incorporated, for example, by modeling the gains as random functions of time (i.e., stochastic processes) $g_{l}^{k}(t), l \in\left\{1,2, \ldots, \operatorname{card}\left(\mathbb{S}_{k}\right)\right\}, k \in\{T, R\}$, each having a time-varying average power.

The envelope and the phase of $H(t ; f)$ are also TFvarying quantities given by $\Xi(t, f) \triangleq|H(t ; f)|$ and $\Psi(t, f) \triangleq$ $\arg \{H(t ; f)\}$. The computation of the distributions of $\Xi(t, f)$ and $\Psi(t, f)$ is not a trivial task, because the term $\left\|\mathbf{p}_{\ell, m}^{S}\right\|$ in (13) is a nonlinear transformation of the random vectors $\widehat{\mathbf{p}}_{\ell}^{T}$ and $\widehat{\mathbf{p}}_{m}^{R}$ (see (11)). However, if the condition $D \gg \max \left\{\left\|\widehat{\mathbf{p}}_{\ell}^{T}\right\|,\left\|\widehat{\mathbf{p}}_{m}^{R}\right\|\right\}$ holds for all $\ell, m$, then this transformation becomes linear and equal to $\left\|\mathbf{p}_{\ell, m}^{S}\right\| \approx D_{\ell}^{T}+D_{m}^{R}$, where $D_{l}^{k}=D / 2+$ $q_{k}\left\langle\mathbf{D}, \widehat{\mathbf{p}}_{l}^{k}\right\rangle / D$, for $l \in\left\{1,2, \ldots, \operatorname{card}\left\{\mathbb{S}_{k}\right\}\right\}, k \in\{T, R\}$, and

$$
q_{k}= \begin{cases}-1, & \text { if } k=T \\ 1, & \text { if } k=R\end{cases}
$$

With this simplification, the FO PDF of $\Xi(t, f)$ can be computed following the procedures presented in $[28,29]$. Thereby, the FO PDF of $\Xi(t, f)$ is given by

$$
\begin{aligned}
& p_{\Xi}(t, f ; \xi)=(2 \pi)^{4} \xi \int_{-\infty}^{\infty}\left|\frac{1}{w}\right| \\
& \cdot \int_{0}^{\infty}\left[\prod_{\ell=1}^{L} \int_{0}^{\infty} p_{g_{\ell}^{T}}\left(c_{\ell}^{T}\right) J_{0}\left(2 \pi c_{\ell}^{T} x_{T}\right) d c_{\ell}^{T}\right] \\
& \cdot J_{0}\left(2 \pi x_{T} w\right) x_{T} d x_{T}
\end{aligned}
$$




$$
\begin{aligned}
& \cdot \int_{0}^{\infty}\left[\prod_{m=1}^{M} \int_{0}^{\infty} p_{g_{m}^{R}}\left(c_{m}^{R}\right) J_{0}\left(2 \pi c_{m}^{R} x_{R}\right) d c_{m}^{R}\right] \\
& \cdot J_{0}\left(\frac{2 \pi x_{R} \xi}{w}\right) x_{R} d x_{R} d w
\end{aligned}
$$

for $\xi \geq 0$ and $t \in\left[0, T_{0}\right]$, where $J_{0}(\cdot)$ is the Bessel function of the first kind and zeroth order. Analogously, the PDF of $\Psi(t, f)$ is given as

$$
p_{\Psi}(t, f ; \psi)=\frac{1}{2 \pi}, \quad 0<\psi \leq 2 \pi, t \in\left[0, T_{0}\right] .
$$

The details on the derivations are omitted for reasons of brevity, but an outline can be found in [30].

We can observe from (21) and (22) that $\Xi(t, f)$ and $\Psi(t, f)$ are FO-stationary random processes, as their PDFs $p_{\Xi}(t, f ; \xi)$ and $p_{\Psi}(t, f ; \psi)$ do not change over time and frequency within the relevant interval $t \in\left[0, T_{0}\right]$. This feature indicates that the nonstationary characteristics of the proposed channel model-if any-are not caused by large-scale factors, such as shadowing or path loss, or by gross changes in the location of the IOs, such as the appearance and disappearance of IOs. We note again that the influence of such large-scale factors has intentionally been neglected in this paper to place emphasis on the nonstationarities caused by the time-varying propagation delays.

Unlike the PDF of the phase $\Psi(t, f)$, which does not depend on the number of multipath components $L \times M$, the PDF of the envelope $\Xi(t, f)$ is highly influenced by the values of $L$ and $M$. Nevertheless, if the gains $g_{l}^{k}$ are Rayleigh distributed r.v., that is, if

$$
p_{g_{l^{k}}}(c)=\frac{2 c}{\left(\rho_{l}^{k}\right)^{2}} \exp \left\{-\frac{c^{2}}{\left(\rho_{l}^{k}\right)^{2}}\right\}, \quad c \geq 0
$$

for $l \in\left\{1,2, \ldots, \operatorname{card}\left(\mathbb{S}_{k}\right)\right\}$ and $k \in\{T, R\}$, where the parameters $\rho_{l}^{k}$ control the dispersion of the Rayleigh distribution, and they are not necessarily equal to each other, then the PDF in (21) can be simplified to

$$
p_{\Xi}(\xi)=\frac{4 \xi}{\sigma_{H}} K_{0}\left(\frac{2 \xi}{\sigma_{H}}\right), \quad \xi \geq 0,
$$

for $t \in\left[0, T_{0}\right]$, where $K_{0}$ is the modified Bessel function of the second kind and zeroth order (see [30] for details on the derivation of (24)). The PDF in (24) is known as the double-Rayleigh distribution [13]. The same result is obtained regardless of the distribution of the gains $g_{l}^{k}$ if the value of $L$ is large (infinitely large in theory).

3.3. Four-Dimensional TF-CF. For the analysis of the correlation properties of $H(t ; f)$, we will consider the following definition of the $4 \mathrm{D}$ TF-CF given in [31, Eq. (3b)]:

$$
R_{H}(t, f ; \Delta t, \Delta f) \triangleq E\left\{H^{*}(t-\Delta t ; f) H(t ; f+\Delta f)\right\} .
$$

Starting from (18) and (25) and assuming that $t_{0}=0$, we show in Appendix A that

$$
\begin{aligned}
& R_{H}(t, f ; \Delta t, \Delta f) \\
& \quad=\sigma_{H} \Upsilon(t, \Delta t) E\left\{\exp \left\{j 2 \pi\left[\Delta t f_{D}\left(\phi_{T}, \phi_{R}\right)\left(\frac{f_{c}+f}{f_{c}}\right)-\Delta f\left(\frac{G_{T}\left(\phi_{T}\right)+G_{S}\left(\phi_{T}, \phi_{R}\right)+G_{R}\left(\phi_{R}\right)}{C_{l}}-t \frac{f_{D}\left(\phi_{T}, \phi_{R}\right)}{f_{c}}\right)\right]\right\}\right\},
\end{aligned}
$$

where $\Upsilon(t, \Delta t)=\Pi_{T_{0}}(t) \Pi_{T_{0}}(t-\Delta t)$, while $\phi_{k}$ is an arbitrary r.v. in $\left\{\phi_{1}^{k}, \phi_{2}^{k}, \ldots, \phi_{\text {card }\left\{\mathbb{S}_{k}\right\}}^{k}\right\}, k \in\{T, R\}$, and

$$
f_{D}\left(\phi_{T}, \phi_{R}\right)=f_{T}\left(\phi_{T}\right)+f_{R}\left(\phi_{R}\right) .
$$

The Doppler frequencies $f_{T}\left(\phi_{T}\right)$ and $f_{R}\left(\phi_{R}\right)$ are equal to

$$
f_{k}\left(\phi_{k}\right)=f_{\max }^{k} \cos \left(\phi_{k}-\gamma_{k}\right), \quad k \in\{T, R\}
$$

where $\gamma_{k}$ is the angle of the velocity vector $\mathbf{v}_{k}$, for $k \in\{T, R\}$, $f_{\max }^{k}=v_{k} / \lambda$, with $v_{k}$ denoting the speed of $T_{X}($ when $k=T)$ and $R_{X}$ (when $\left.k=R\right)$. In turn, the function $G_{S}\left(\phi_{T}, \phi_{R}\right)$ in (26) is given as

$$
\begin{aligned}
G_{S} & \left(\phi_{T}, \phi_{R}\right) \\
& =\left\|\mathbf{D}-G_{T}\left(\phi_{T}\right) \mathbf{u}_{T}\left(\phi_{T}\right)+G_{R}\left(\phi_{R}\right) \mathbf{u}_{R}\left(\phi_{R}\right)\right\|,
\end{aligned}
$$

where $\mathbf{u}_{k}\left(\phi_{k}\right)$ is a unit vector that points at the direction specified by $\phi_{k}$, for $k \in\{T, R\}$. Invoking the expected value theorem, we can express $R_{H}(t, f ; \Delta t, \Delta f)$ as

$$
\begin{aligned}
& R_{H}(t, f ; \Delta t, \Delta f)=\sigma_{H} \Upsilon(t, \Delta t) \\
& \quad \cdot \int_{-\pi}^{\pi} \int_{-\pi}^{\pi} \exp \left\{j 2 \pi\left[\Delta t f_{D}\left(\phi_{T}, \phi_{R}\right)\left(\frac{f_{c}+f}{f_{c}}\right)-\Delta f\left(\frac{G_{T}\left(\phi_{T}\right)+G_{S}\left(\phi_{T}, \phi_{R}\right)+G_{R}\left(\phi_{R}\right)}{C_{l}}-t \frac{f_{D}\left(\phi_{T}, \phi_{R}\right)}{f_{c}}\right)\right]\right\} p_{\phi}^{T}\left(\phi_{T}\right) \\
& \quad \cdot p_{\phi}^{R}\left(\phi_{R}\right) d \phi_{R} d \phi_{T} .
\end{aligned}
$$


The result in (30) can be simplified to the product of two line integrals if the condition $D \gg \max \left\{G_{T}\left(\phi_{T}\right), G_{R}\left(\phi_{R}\right)\right\}$ holds. Under this condition,

$$
G_{S}\left(\phi_{T}, \phi_{R}\right) \approx D_{T}\left(\phi_{T}\right)+D_{R}\left(\phi_{R}\right)
$$

where

$$
D_{k}\left(\phi_{k}\right)=\frac{D}{2}+q_{k} G_{k}\left(\phi_{k}\right) \frac{\left\langle\mathbf{D}, \mathbf{u}_{k}\left(\phi_{k}\right)\right\rangle}{D}, \quad k \in\{T, R\} .
$$

Thereby, we have

$$
R_{H}(t, f ; \Delta t, \Delta f) \approx R_{H}^{T}(t, f ; \Delta t, \Delta f) R_{H}^{R}(t, f ; \Delta t, \Delta f)
$$

with

$$
\begin{aligned}
& R_{H}^{k}(t, f ; \Delta t, \Delta f)=\zeta_{\mathrm{k}} \Upsilon(t, \Delta t) \\
& \cdot \int_{-\pi}^{\pi} \exp \left\{j 2 \pi \left[\Delta t f_{k}\left(\phi_{k}\right)\left(\frac{f_{c}+f}{f_{c}}\right)\right.\right. \\
& \left.\left.-\Delta f\left(\frac{G_{k}\left(\phi_{k}\right)+D_{k}\left(\phi_{k}\right)}{C_{l}}-t \frac{f_{k}\left(\phi_{k}\right)}{f_{c}}\right)\right]\right\} \\
& \cdot p_{\phi}^{k}\left(\phi_{k}\right) d \phi_{k}, \quad k \in\{T, R\} .
\end{aligned}
$$

Equations (33) and (34) show that the 4D TF-CF of the proposed channel model can be factorized as the Kronecker product (tensor) of two different TF-CFs, one due to the signal's dispersion at the transmitter side $\left(R_{H}^{T}(t, f ; \Delta t, \Delta f)\right)$ and the other to the dispersion at the receiver side $\left(R_{H}^{R}(t, f ; \Delta t, \Delta f)\right)$. This is not a novel result, nevertheless, as it has been shown in previous papers that the channel's correlation function can be expressed by the Kronecker product if the transmitted signal arrives at the receiver via double interactions with IOs $[32,33]$. However, to the best of the authors' knowledge, the GBSMs that have been proposed for doubly and triply selective V2V channels assuming the propagation of plane waves and a double interaction with IOs fulfill the WSSUS condition by design [24-26]. In contrast, we can observe from (26), (30), (33), and (34) that the proposed channel model is a non-WSSUS random process, because its $4 \mathrm{D}$ TF-CF is a TF-varying function, meaning that $R_{H}\left(t_{1}, f_{1} ; \Delta t, \Delta f\right) \neq R_{H}\left(t_{2}, f_{2} ; \Delta t, \Delta f\right)$ for different observation instants $\left(t_{1}, f_{1}\right)$ and $\left(t_{2}, f_{2}\right)$. We recall that a TFdispersive channel is said to fulfill the WSSUS condition if its mean value is a constant, and its TF-CF is invariant over the time $t$ and the frequency $f$ variables, that is, if
$R_{H}\left(t_{1}, f_{1} ; \Delta t, \Delta f\right)=R_{H}\left(t_{2}, f_{2} ; \Delta t, \Delta f\right)$, for $\left(t_{1}, f_{1}\right) \neq\left(t_{2}, f_{2}\right)$ $[5,34]$. In addition, we recall that the fulfillment of the WSSUS condition implies that the channel is simultaneously WSS in the time and the frequency domains [5].

The nonstationary features of our channel model are noteworthy because our modeling framework does not consider macroscopic (large-scale) or microscopic (small-scale) factors that are already well-known sources of nonstationarities, such as path loss, shadowing, the appearance and disappearance of IOs, or time-varying AODs and AOAs. The nonstationarities of the channel model defined by (3) stem from the propagation delays' temporal variations. This microscopic source of nonstationarities has passed practically unnoticed in the literature, and its analysis has therefore received little attention.

Equation (30) shows that the time dependence of $R_{H}(t, f ; \Delta t, \Delta f)$ is given by the factor $t \cdot \Delta f \cdot f_{D}\left(\phi_{T}, \phi_{R}\right) / f_{c}$, whereas its frequency dependence is given by $f \cdot \Delta t$. $f_{D}\left(\phi_{T}, \phi_{R}\right) / f_{c}$. These two factors must be equal to zero in order for the proposed channel model to be WSS in both the time $t$ and the frequency $f$ variables (i.e., to enforce the WSSUS condition). Hence, this condition can only be met if the Doppler frequency $f_{D}\left(\phi_{T}, \phi_{R}\right)$ is equal to zero, meaning that the channel is static. Nevertheless, a static channel model is of little relevance for the analysis of $\mathrm{V} 2 \mathrm{~V}$ communication systems.

While the proposed channel model does not fulfill the WSSUS condition, it is worth noting that $R_{H}(t, f ; \Delta t, \Delta f)$ becomes a time-independent function over the observation region defined by $\Upsilon(t, \Delta t)$ if we make $\Delta f=0$. For that particular case, $\left.R_{H}(t, f ; \Delta t ; \Delta f)\right|_{\Delta f=0}=\Upsilon(t, \Delta t) T_{H}(f ; \Delta t)$, where $T_{H}(f ; \Delta t)$ is a frequency-varying time correlation function (TCF) given as

$$
\begin{aligned}
T_{H} & (f ; \Delta t) \\
& =\sigma_{H} \int_{-\pi}^{\pi} \int_{-\pi}^{\pi} \exp \left\{j 2 \pi \Delta t f_{D}\left(\phi_{T}, \phi_{R}\right)\left(\frac{f_{c}+f}{f_{c}}\right)\right\} \\
& \cdot p_{\phi}^{T}\left(\phi_{T}\right) p_{\phi}^{R}\left(\phi_{R}\right) d \phi_{R} d \phi_{T} .
\end{aligned}
$$

This means that, for a constant value of $f$, say $f=f^{\prime}$, the channel transfer function $H\left(t ; f^{\prime}\right)$ can be deemed WSS in the time domain within the relevant observation interval $t \in\left[0, T_{0}\right]$. Analogously, $R_{H}(t, f ; \Delta t, \Delta f)$ can be simplified to a frequency-independent function if $\Delta t=0$. Under such conditions, $\left.R_{H}(t, f ; \Delta t ; \Delta f)\right|_{\Delta t=0}=\Pi_{T_{0}}(t) F_{H}(t ; \Delta f)$, where $F_{H}(t ; \Delta f)$ is a time-varying frequency correlation function (FCF) equal to

$$
F_{H}(t ; \Delta f)=\sigma_{H} \int_{-\pi}^{\pi} \int_{-\pi}^{\pi} \exp \left\{-j 2 \pi \Delta f\left[\frac{G_{T}\left(\phi_{T}\right)+G_{S}\left(\phi_{T}, \phi_{R}\right)+G_{R}\left(\phi_{R}\right)}{C_{l}}-t \frac{f_{D}\left(\phi_{T}, \phi_{R}\right)}{f_{c}}\right]\right\} p_{\phi}^{T}\left(\phi_{T}\right) p_{\phi}^{R}\left(\phi_{R}\right) d \phi_{R} d \phi_{T} .
$$

Thus, for a constant value of $t$, say $t=t^{\prime}$, the channel transfer function $H\left(t^{\prime} ; f\right)$ can be modeled as a WSS process in the frequency domain. We note that the
Kronecker factorization holds also for both the frequencyvarying TCF $T_{H}(f ; \Delta t)$ and the time-varying $\mathrm{FCF}$ $F_{H}(t ; \Delta f)$. 
Even though the proposed channel model is a nonWSSUS random process, the two particular cases discussed above show that $H(t ; f)$ fulfills the WSS condition in one dimension (either time or frequency) if the channel's dispersion is neglected in the other dimension. This characteristic makes our proposal compatible with some notable channel models that are widely accepted benchmarks for the performance analysis of wireless communication systems. Consider, for example, the model proposed by Clarke in [9] for WSS frequency-nonselective (narrowband) Rayleigh fading channels.

The mathematical definition of wide-sense stationarity requires the first- and second-order (SO) statistical properties of a random process to be invariant over the corresponding index set (the time or frequency lines, or the TF plane). While difficult to meet, the condition of statistical invariance should always be the yardstick to determine whether a random process is WSS or not. Then, if the process is found to be nonstationary, we should turn our attention to the concept of quasi-stationarity to obtain a less rigid notion of its stationarity properties [35]. Even though a thorough analysis of quasi-stationarity is beyond the scope of this paper, some interesting remarks can be made in that regard from (26). For example, in practice, the values of $f$ are restricted to $f \in[-B / 2, B / 2]$, where $B$ is the signal's bandwidth. Given that $B$ is typically much smaller than the carrier frequency $f_{c}$, the factor $f \cdot \Delta t \cdot f_{D}\left(\phi_{T}, \phi_{R}\right) / f_{c}$, which determines the dependence of $R_{H}(t, f ; \Delta t, \Delta f)$ on the frequency variable $f$, can be approximated as $\Delta t \cdot f_{D}\left(\phi_{T}, \phi_{R}\right) f / f_{c} \approx 0$. This means that the channel can be modeled by a quasi-WSS random process in the frequency domain if we consider a frequency observation interval of a length similar to the signal's bandwidth and $B \ll f_{c}$. On the other hand, the dependence of $R_{H}(t, f ; \Delta t, \Delta f)$ on the time variable $t$ is given by the term $t \cdot \Delta f \cdot f_{D}\left(\phi_{T}, \phi_{R}\right) / f_{c}$, which is influenced by the degree of mobility of $T_{X}$ and $R_{X}$ (through the Doppler frequency shift $f_{D}\left(\phi_{T}, \phi_{R}\right)$ ), the signal's bandwidth (through the frequency lag $\Delta f \in[-B, B])$, and the carrier frequency $f_{c}$. Hence, the channel may be modeled by a quasi-WSS random process in the time domain over the region associated with $\Upsilon(t, \Delta t)$ if the MSs move at very low speeds (such that $f_{D}\left(\phi_{T}, \phi_{R}\right) \approx 0$ ), or if the signal's bandwidth $B$ is much smaller than $f_{c}$, such that $f_{D}\left(\phi_{T}, \phi_{R}\right) \Delta f / f_{c} \approx 0, \forall \Delta f \in$ $[-B, B]$. Nonetheless, to properly assess the quasi-stationarity properties of the proposed channel model, a more formal and mathematically rigorous analysis is required.

3.4. TF-Dependent Delay and Doppler Profiles. The spectral properties of the proposed channel model can be analyzed on the grounds of the TF-dependent delay and Doppler profiles. These two functions are given, respectively, as [31]

$$
\begin{aligned}
& P_{H}(t, f ; \tau) \triangleq \int_{-\infty}^{\infty} R_{H}(t, f ; 0, \Delta f) \exp \{j 2 \pi \tau \Delta f\} d \Delta f \\
& =\Pi_{T_{0}}(t) \int_{-\infty}^{\infty} F_{H}(t ; \Delta f) \exp \{j 2 \pi \tau \Delta f\} d \Delta f
\end{aligned}
$$

$$
\begin{aligned}
& D_{H}(t, f ; v) \\
& \quad \triangleq \int_{-\infty}^{\infty} R_{H}(t, f ; \Delta t, 0) \exp \{-j 2 \pi v \Delta t\} d \Delta t \\
& =\Pi_{T_{0}}(t) \\
& \quad \cdot \int_{-\infty}^{\infty} \Pi_{T_{0}}(t-\Delta t) T_{H}(f ; \Delta t) \exp \{-j 2 \pi v \Delta t\} d \Delta t .
\end{aligned}
$$

By substituting (36) and (35) into (38) and (40), we find

$$
\begin{aligned}
& P_{H}(t, f ; \tau)=\sigma_{H} \Pi_{T_{0}}(t) \int_{-\pi}^{\pi} \int_{-\pi}^{\pi} \delta\left(\tau+t \frac{f_{D}\left(\phi_{T}, \phi_{R}\right)}{f_{c}}\right. \\
& \left.-\frac{G_{T}\left(\phi_{T}\right)+G_{S}\left(\phi_{T}, \phi_{R}\right)+G_{R}\left(\phi_{R}\right)}{C_{l}}\right) p_{\phi}^{T}\left(\phi_{T}\right) \\
& \cdot p_{\phi}^{R}\left(\phi_{R}\right) d \phi_{R} d \phi_{T} \\
& D_{H}(t, f ; v)=\sigma_{H} T_{0} \Pi_{T_{0}}(t) \\
& \cdot \int_{-\pi}^{\pi} \int_{-\pi}^{\pi} \operatorname{sinc}\left(\pi T_{0}\left[v-f_{D}\left(\phi_{T}, \phi_{R}\right)\left(\frac{f_{c}+f}{f_{c}}\right)\right]\right) \\
& \cdot \exp \left\{-j 2 \pi\left[v-f_{D}\left(\phi_{T}, \phi_{R}\right)\left(\frac{f_{c}+f}{f_{c}}\right)\right]\left(t-\frac{T_{0}}{2}\right)\right\} \\
& \cdot p_{\phi}^{T}\left(\phi_{T}\right) p_{\phi}^{R}\left(\phi_{R}\right) d \phi_{R} d \phi_{T},
\end{aligned}
$$

where $\operatorname{sinc}(x) \triangleq \sin (x) / x$ is the sinc function. Equation (41) shows that the delay profile of the proposed channel model is a frequency-invariant function $P_{H}(t ; \tau)$, which depends on the channel's angular statistics and the propagation area geometry. The Doppler profile, on the other hand, is a TF-dependent function that depends only on the channel's angular statistics.

\section{Particular Case: The Geometrical Two-Ring Scattering Model}

4.1. Background. The definite integrals in (30), (33), and (34) can be evaluated numerically without major difficulties if $G_{T}, G_{R}, p_{\phi}^{T}$, and $p_{\phi}^{R}$ are known. In fact, the $4 \mathrm{D}$ TF-CF can be expressed in a closed form if the abovementioned functions allow writing the integral at the right-hand side of (34) in terms of standard functions. This is demonstrated in this section by considering the particular case of the geometrical two-ring scattering model and the von Mises distribution of the AOD and AOA. The geometrical two-ring model has widely been employed as a reference to analyze the correlation properties of F2M and V2V fading channels assuming the fulfillment of the WSSUS condition [24, 33]. In this geometrical model, $T_{X}$ and $R_{X}$ are each surrounded by a ring of IOs, in such a way that

$$
\begin{aligned}
& G_{T}\left(\phi_{T}\right)=r_{T}, \quad \forall \phi_{T} \\
& G_{R}\left(\phi_{R}\right)=r_{R}, \quad \forall \phi_{R},
\end{aligned}
$$

where $r_{k}, k \in\{T, R\}$, is the radius of the ring of IOs surrounding $T_{X}$ (when $k=T$ ) and $R_{X}$ (when $k=R$ ). An illustration of this model is shown in Figure 2. 


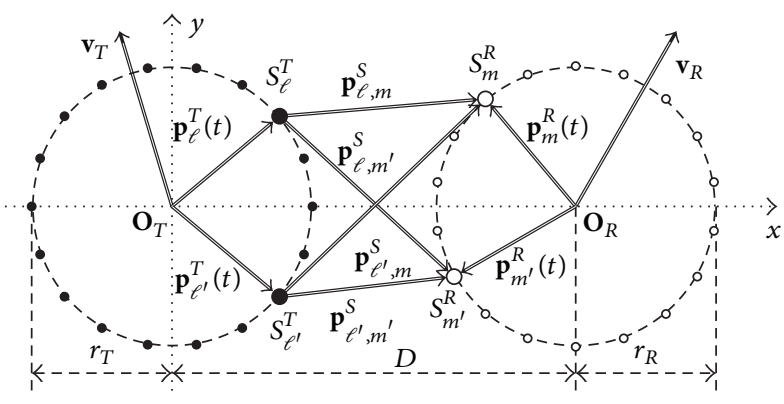

FIGURE 2: The geometrical two-ring model.

The von Mises distribution, on the other hand, is a well-known model for the statistics of circular data and is analogous to the Gaussian distribution on the line [36]. Following the von Mises distribution, we define

$$
p_{\phi}^{k}(\phi)=\frac{\exp \left\{\kappa_{k} \cos \left(\phi-\mu_{k}\right)\right\}}{2 \pi I_{0}\left(\kappa_{k}\right)}, \quad k \in\{T, R\},
$$

where $\mu_{k}$ and $\kappa_{k}$ are the distribution's mean value and concentration parameter and $I_{0}$ is the modified Bessel function of the first kind and zeroth order.

4.2. Closed-Form Solution of the 4D TF-CF. Without loss of generality, we will assume that the time-invariant vectors $\mathbf{O}_{T}$ and $\mathbf{O}_{R}$ introduced in Figure 1 are collinear with the $x$ axis, such that $\mathbf{D}=\mathbf{O}_{R}-\mathbf{O}_{T}=[D, 0]^{\dagger}$. In addition, we will assume that $D \gg \max \left\{r_{T}, r_{R}\right\}$. Under these conditions, and substituting (43a), (43b), and (44) into (34), we show in Appendix B that

$$
\begin{aligned}
& R_{H}(t, f ; \Delta t, \Delta f)=\sigma_{H} \Upsilon(t, \Delta t) \\
& \cdot \frac{\exp \left\{-j 2 \pi \Delta f\left(D+r_{T}+r_{R}\right) / C_{l}\right\}}{I_{0}\left(\kappa_{T}\right) I_{0}\left(\kappa_{R}\right)} \\
& \cdot I_{0}\left(\left\{\left[\kappa_{T} \cos \left(\mu_{T}\right)+j 2 \pi B_{c}^{T}\right]^{2}\right.\right. \\
& \left.\left.+\left[\kappa_{T} \sin \left(\mu_{T}\right)+j 2 \pi B_{s}^{T}\right]^{2}\right\}^{1 / 2}\right) \\
& \cdot I_{0}\left(\left\{\left[\kappa_{R} \cos \left(\mu_{R}\right)+j 2 \pi B_{c}^{R}\right]^{2}\right.\right. \\
& \left.\left.+\left[\kappa_{R} \sin \left(\mu_{R}\right)+j 2 \pi B_{s}^{R}\right]^{2}\right\}^{1 / 2}\right)
\end{aligned}
$$

where

$$
\begin{aligned}
B_{c}^{k} & =f_{\max }^{k} \cos \left(\gamma_{k}\right) Z(t, f, \Delta t, \Delta f)-q_{k} \frac{\Delta f r_{k}}{C_{l}} \\
B_{s}^{k} & =f_{\max }^{k} \sin \left(\gamma_{k}\right) Z(t, f, \Delta t, \Delta f)
\end{aligned}
$$

for $k \in\{T, R\}$, and

$$
Z(t, f, \Delta t, \Delta f)=\Delta t\left(\frac{f_{c}+f}{f_{c}}\right)+\Delta f \frac{t}{f_{c}} .
$$

4.3. Numerical Examples. In the remainder of this section, we present some graphical examples of the 4D TF-CF $R_{H}(t, f ; \Delta t, \Delta f)$ in (45) and the corresponding time-varying delay profile $P_{H}(t ; \tau)$ and TF-dependent Doppler profile $D_{H}(t, f ; v)$. The expressions presented in Section 3 for the FO PDFs of the envelope and phase are not considered here because they have already been analyzed in [30]. For the evaluation of $R_{H}(t, f ; \Delta t, \Delta f), P_{H}(t ; \tau)$, and $D_{H}(t, f ; v)$, we have chosen $f_{c}=5.9 \mathrm{GHz}, B=10 \mathrm{MHz}, T_{0}=6.4 \mathrm{~ms}, \gamma_{T}=60^{\circ}$, $\gamma_{R}=250^{\circ}, D=500 \mathrm{~m}, r_{T}=30 \mathrm{~m}, r_{R}=40 \mathrm{~m}, f_{\max }^{T}=500 \mathrm{~Hz}$ (corresponding to a speed of $91.4 \mathrm{~km} / \mathrm{h}$ ), $f_{\max }^{R}=500 \mathrm{~Hz}$, $\sigma_{H}=1, \kappa_{T}=1$ (moderate nonisotropic dispersion), $\kappa_{R}=10$ (highly nonisotropic dispersion), $\mu_{T}=60^{\circ}$, and $\mu_{R}=120^{\circ}$. The system related parameters $f_{c}, B$, and $T_{0}$ are taken from the IEEE 802.11p standard for vehicular DSRC systems [3] (the value of $T_{0}$ equals the duration of a signal frame comprising 800 data symbols, each having a length of $8 \mu \mathrm{s}$ ).

4.3.1. $4 D$ TF-CF. A 3D graph and a contour plot of the absolute value of $R_{H}(t, f ; \Delta t, \Delta f)$ are presented in Figure 3 for an arbitrary observation point $(t, f)=\left(0.5 T_{0}, 0.3 B\right)$ in the TF plane. These two graphs show that the proposed channel model is a non-WSSUS random process, because its $4 \mathrm{D}$ TF-CF has an asymmetrical shape. We recall that a fundamental property of WSS random processes is that their autocorrelation function (ACF) is symmetric around the origin [37, Theorem 10.12]. Particularly, for a complex-valued one-dimensional (1D) random process $\chi(\eta)$ defined in either the time or the frequency domain, the WSS condition implies that $R_{\chi}^{*}(\eta ; \Delta \eta)=R_{\chi}(\eta ;-\Delta \eta), \forall \eta, \Delta \eta \in \mathbb{R}$, where $R_{\chi}(\eta ; \Delta \eta) \triangleq$ $E\left\{\chi^{*}(\eta) \chi(\eta-\Delta \eta)\right\}$ is the ACF of $\chi(\eta)$. Analogously, for a complex-valued $2 \mathrm{D}$ random process $\chi(t ; f)$ defined in the TF plane, it can be shown that the WSSUS condition implies that $R_{\chi}(t, f ; \Delta t, \Delta f)=R_{\chi}^{*}(t, f ; \Delta t,-\Delta f)=R_{\chi}^{*}(t, f ;-\Delta t, \Delta f)=$ $R_{\chi}(t, f ;-\Delta t,-\Delta f), \forall t, f$, where $R_{\chi}(t, f ; \Delta t, \Delta f)$ is the 4D TFCF defined in (25).

To make the asymmetries of $R_{H}(t, f ; \Delta t, \Delta f)$ more evident, Figure 4 shows curves of the absolute value of $R_{H}(t, f ; \Delta t, \Delta f)$ generated for fixed values of either the frequency lag $\Delta f$ or the time lag $\Delta t$. The curves shown in Figure 4(a) were computed for $\Delta f \in\left\{-2.5 \times 10^{6},-1.25 \times\right.$ $\left.10^{6}, 0,1.25 \times 10^{6}, 2.5 \times 10^{6}\right\}$, while the ones presented in Figure $4(\mathrm{~b})$ were generated for $\Delta t \in\left\{-8 \times 10^{-4},-3 \times\right.$ $\left.10^{-4}, 0,3 \times 10^{-4}, 8 \times 10^{-4}\right\}$. The graphs obtained for $\Delta f=0$ and $\Delta t=0$ correspond to the particular cases of the frequencyvarying TCF $T_{H}(f ; \Delta t)$ and the time-varying $\operatorname{FCF} F_{H}(t ; \Delta f)$, respectively.

Aside from the particular cases of $T_{H}(f ; \Delta t)$ and $F_{H}(t ; \Delta f)$, we can observe from Figure 4 that $\left|R_{H}(t, f ; \Delta t, \Delta f)\right|$ is an asymmetrical function. However, the asymmetries of $\left|R_{H}(t, f ; \Delta t, \Delta f)\right|$ smoothen off gradually as the channel becomes nondispersive in one dimension. This behavior shows that the proposed channel model's quasi-stationary characteristics are stronger as the 2D TF lag $(\Delta t, \Delta f)$ approaches the ideal pairings that lead to $T_{H}(f ; \Delta t)$ and $F_{H}(t ; \Delta f)$. On the other hand, the symmetrical shape of $T_{H}(f ; \Delta t)$ and $F_{H}(t ; \Delta f)$ can be taken as graphical evidence for the observation we made in the previous section about the 


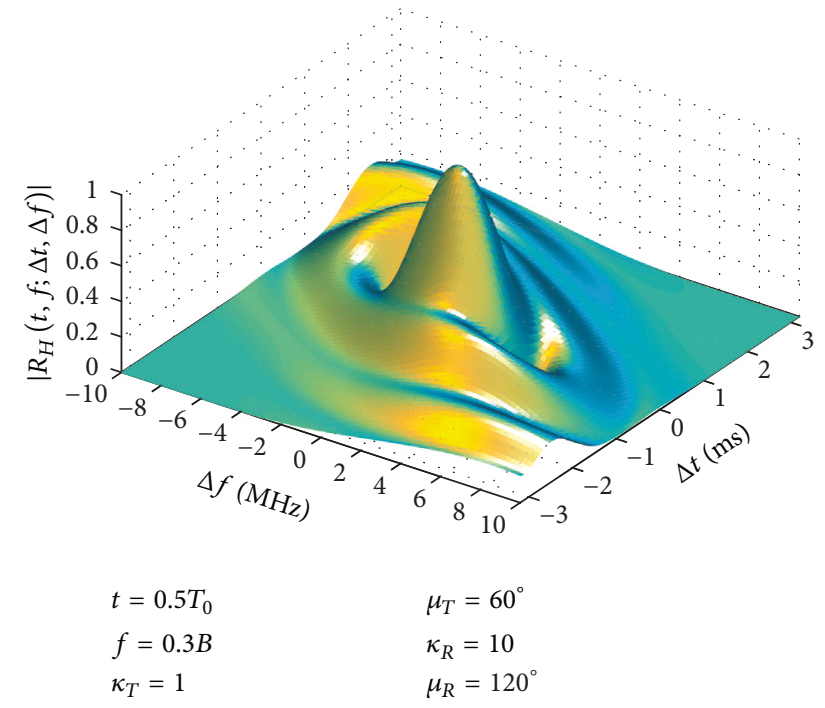

(a) $3 \mathrm{D}$ surface

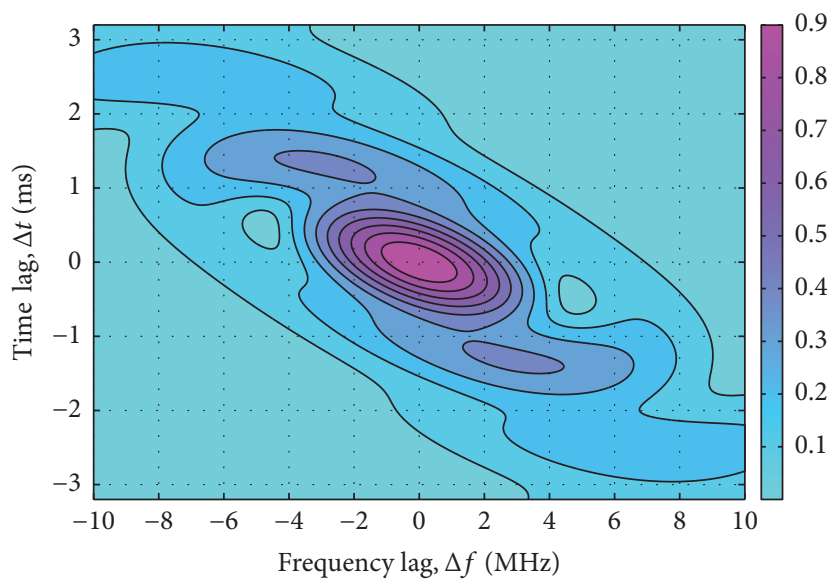

(b) Contour

Figure 3: Absolute value of the $4 \mathrm{D}$ TF-CF $R_{H}(t, f ; \Delta t, \Delta f)$ at $(t, f)=\left(0.5 T_{0}, 0.3 B\right\}$.

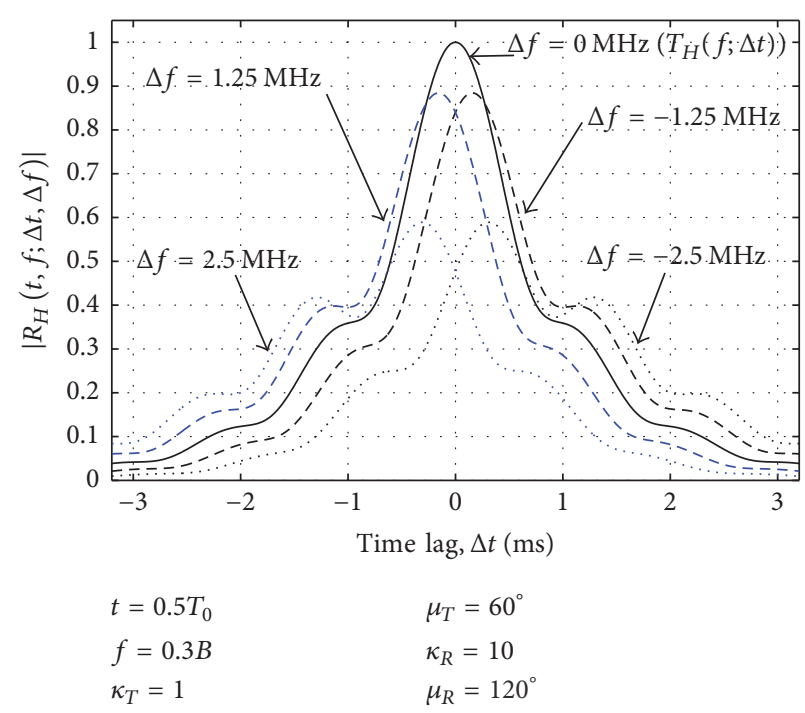

(a) Constant values of the frequency lag $\Delta f$

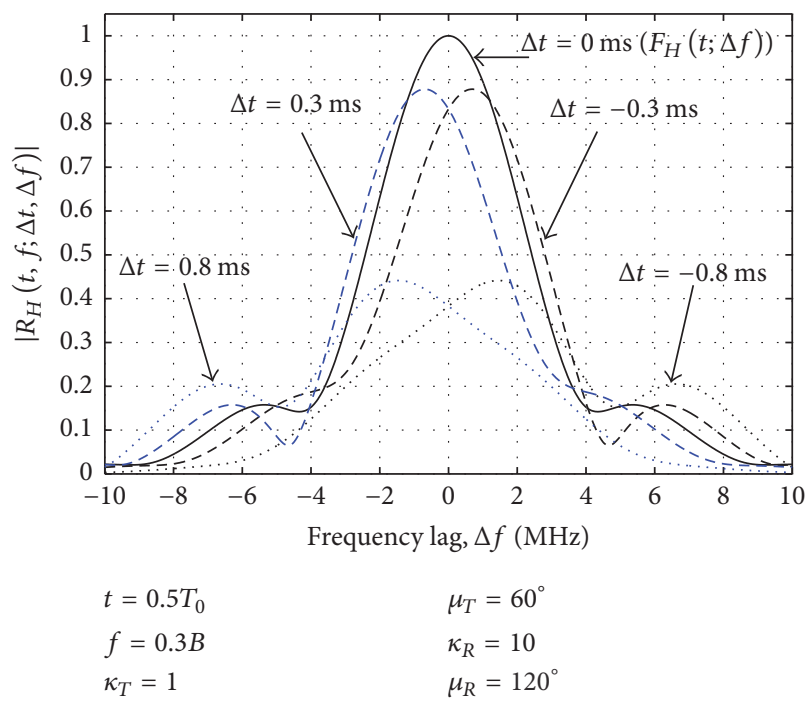

(b) Constant values of the time lag $\Delta t$

Figure 4: Absolute value of the 4D TF-CF $R_{H}(t, f ; \Delta t, \Delta f)$ at $(t, f)=\left(0.5 T_{0}, 0.3 B\right\}$.

proposed channel model becoming a WSS random process in one dimension (time or frequency) if its dispersiveness is neglected in the other dimension. Even though these two ideal cases have limited practical significance, they are of theoretical relevance, as they allow identifying scenarios where the WSS condition is met in one dimension.

4.3.2. Time-Frequency Dependent Delay Profile. A 3D graph and a contour plot of the time-varying delay profile $P_{H}(t ; \tau)$ are presented in Figure 5. To highlight the time-varying nature of $P_{H}(t ; \tau)$, we have considered a larger observation time window of length $T_{0}=320 \mathrm{~ms}$. In our simulation setup, the MSs are moving towards each other along nearly parallel trajectories. This explains the shortening of the minimum propagation delay, $\tau_{\min }(t)$, which is defined as the propagation delay of the plane wave that travels to $R_{X}$ over the shortest path. Note that $\tau_{\min }(t)$ shrinks from $1.6678 \mu \mathrm{s}$ at $t=0$ to $1.6457 \mu \mathrm{s}$ at $t=320 \mathrm{~ms}$. On the other hand, the maximum propagation delay, $\tau_{\max }(t)$, which is defined as the propagation delay of the plane wave that travels to $R_{X}$ over the largest path, increases from $2.1127 \mathrm{~ms}$ to around $2.15 \mathrm{~ms}$. This increment is due to the fact that as time evolves, the MSs get closer to some IOs but farther off from others. 


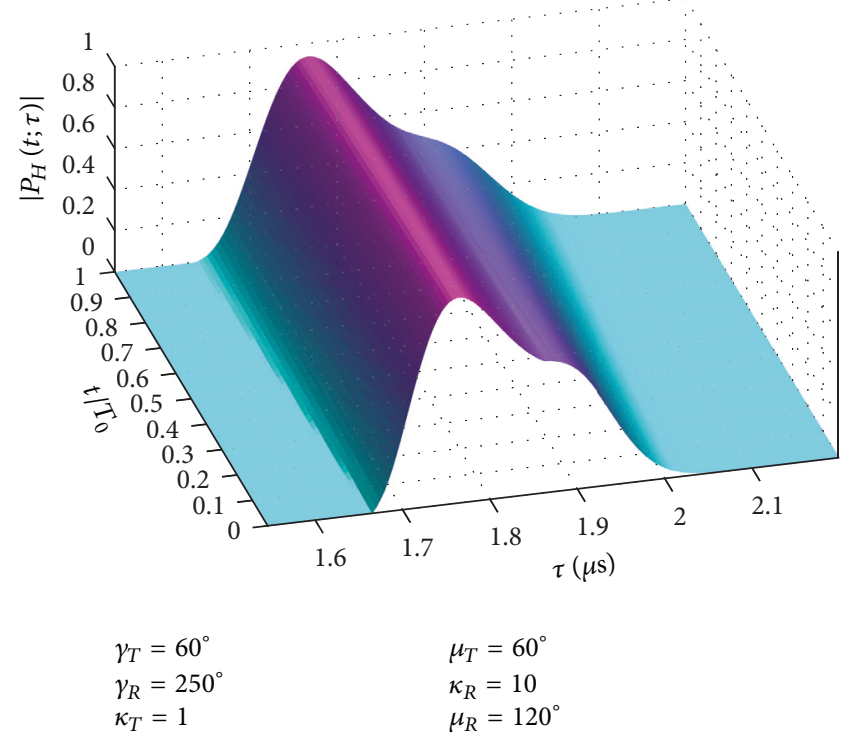

(a) $3 \mathrm{D}$ surface

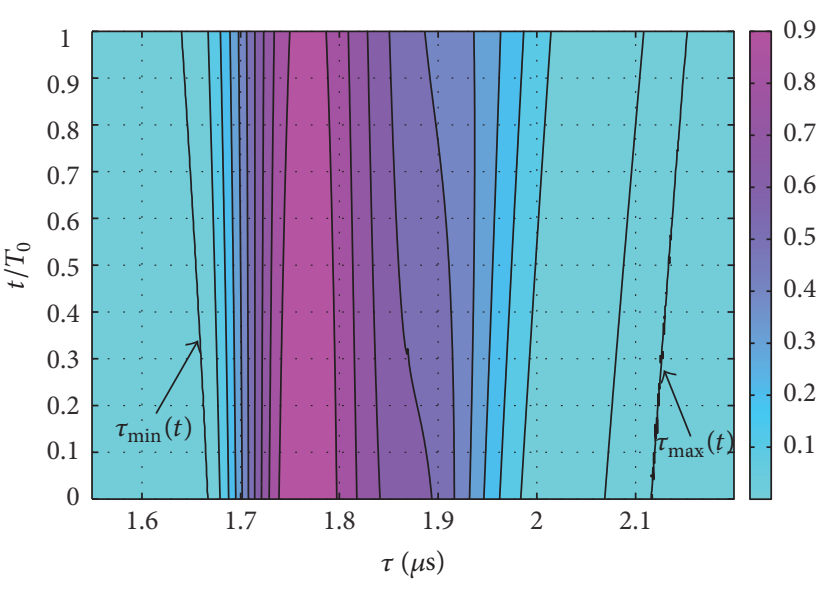

(b) Contour

FIgURE 5: Absolute value of the time-varying delay profile $P_{H}(t ; \tau)$ for $T_{0}=320 \mathrm{~ms}$.

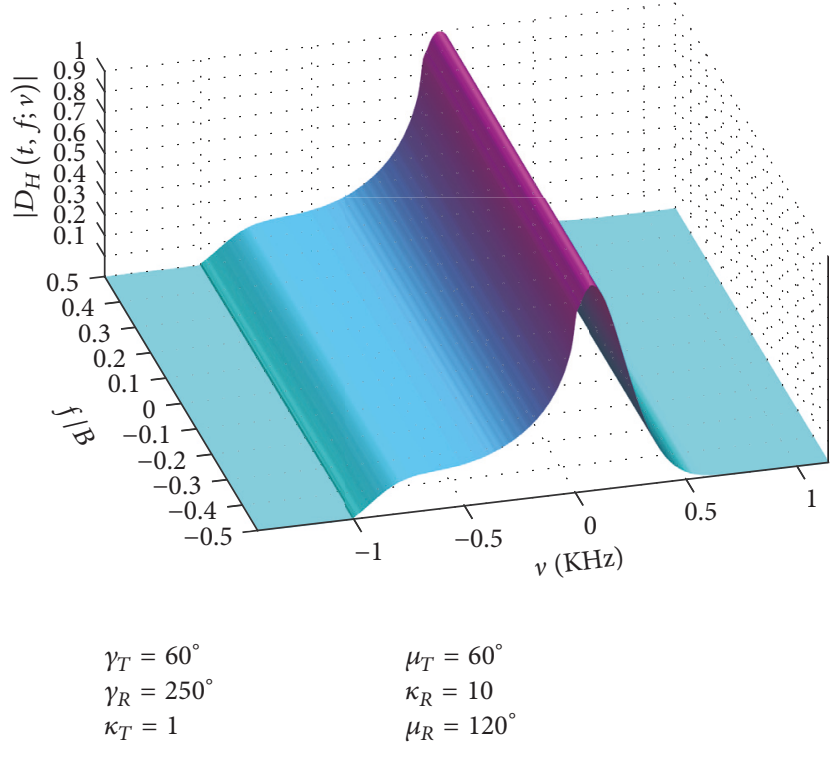

(a) $3 \mathrm{D}$ surface

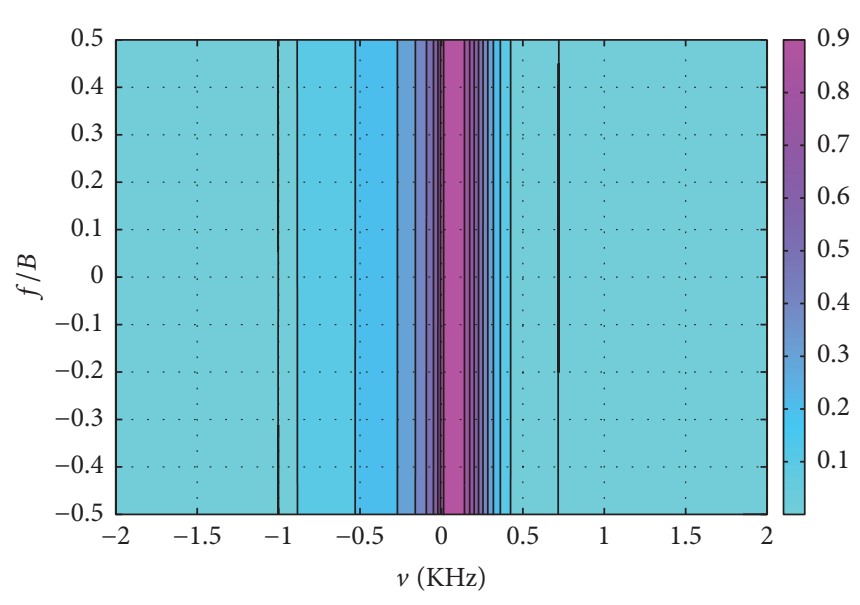

(b) Contour plot

Figure 6: Absolute value of the TF-varying Doppler profile $D_{H}(t, f ; v)$ for $t=0.8 T_{0}$ and $T_{0}=320 \mathrm{~ms}$.

4.3.3. Time-Frequency Dependent Doppler Profile. Finally, Figure 6 shows a 3D graph and a contour plot of the TFvarying Doppler profile $D_{H}(t, f ; v)$ evaluated at $t=0.8 T_{0}$. While the variability of $D_{H}(t, f ; v)$ in the frequency $f$ variable is not evident in the figure, the shape of $D_{H}(t, f ; v)$ does vary in the frequency domain, but at a rate that is much smaller than the length of the observation window $f \in[-B / 2, B / 2]$. This is demonstrated in Figure 7, where we present the same contour plot of Figure 6(b) but for an expanded observation window $f \in[-100 B, 100 B]$. Regarding the time variations of $D_{H}(t, f ; v)$, these are the result of convolving the spectra of the time-invariant TCF $T_{H}(f ; \Delta t)$ and the time-dependent window function $\Pi_{T_{0}}(t)$. Such temporal variations manifest themselves by a rippling effect in the spectrum of $T_{H}(f ; \Delta t)$. Our numerical experiments indicate that this effect is rather mild, making the time-varying nature of $D_{H}(t, f ; v)$ almost imperceptible. This is demonstrated by comparing the $3 \mathrm{D}$ surface of Figure 6(a) with the one presented in Figure 8, where the value of the observation time $t$ is equal to $t=0.1 T_{0}$. Hence, based on our numerical results, we conclude that, for most practical purposes, the Doppler profile of the proposed channel model can be considered a time-invariant function 


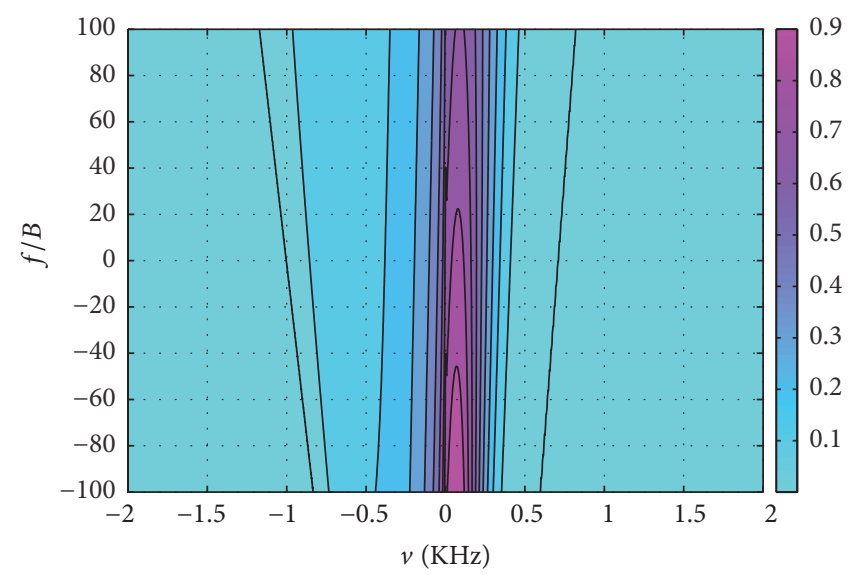

Figure 7: Contour plot of the absolute value of the TF-varying Doppler profile $D_{H}(t, f ; v)$ for $t=0.8 T_{0}, T_{0}=320 \mathrm{~ms}$, and an extended observation window in the frequency domain.

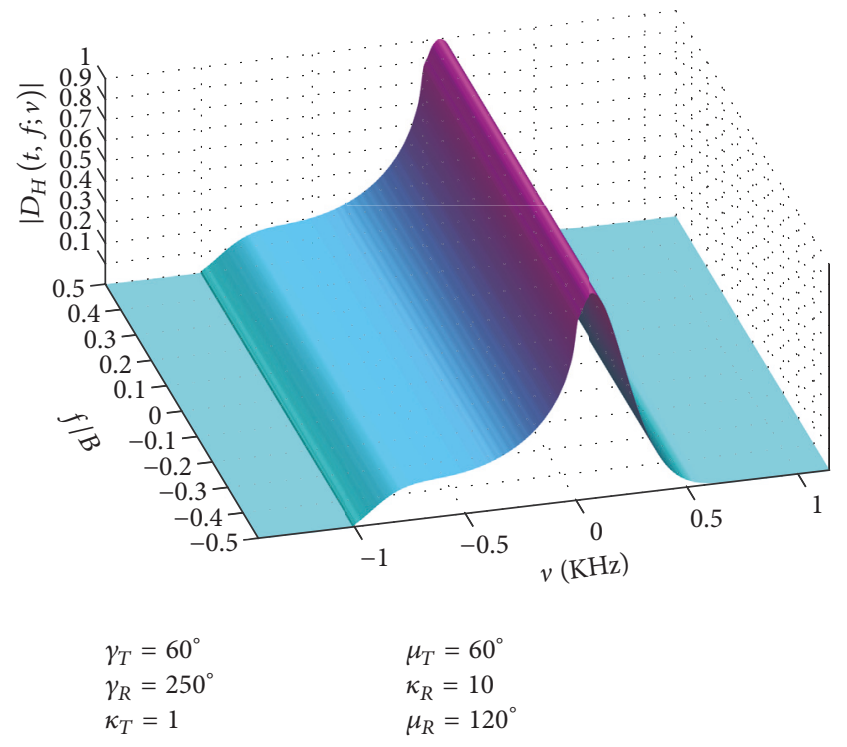

FIGURE 8: 3D surface of the absolute value of the TF-varying Doppler profile $D_{H}(t, f ; v)$ for $t=0.1 T_{0}$ and $T_{0}=320 \mathrm{~ms}$.

$D_{H}(f ; v)$. However, the frequency variations of $D_{H}(f ; v)$ should in general not be neglected.

\section{Conclusions}

In this paper, we have proposed a novel GBSM for smallscale non-WSSUS V2V double-Rayleigh fading channels. The model is quite flexible and can easily be adapted to a wide range of $2 \mathrm{D}$ and $3 \mathrm{D}$ geometrical propagation scenarios. Based on this model, we derived general expressions for the envelope and phase distributions, the $4 \mathrm{D}$ TF-CF, and the TF-dependent delay and Doppler profiles. The obtained expressions show that the 4D TF-CF of the proposed channel model is a TF-varying function. However, such expressions also show that the corresponding delay profile is invariant in the frequency domain, while the Doppler profile can be deemed invariant in the time domain for most practical purposes. Even though the proposed model does not fulfill the WSS condition simultaneously in the time and the frequency domains, it becomes a WSS random process in one dimension (time or frequency) if the channel's dispersiveness is neglected in the other dimension. For the more relevant case of $2 \mathrm{D}$ dispersion, our results suggest that the proposed channel model is quasi-WSS over small observation regions in the TF plane. However, a more formal analysis is necessary to assess the model's quasi-stationarity characteristics.

\section{Appendix}

\section{A. Derivation of (26)}

By a direct evaluation of (25), and taking into account the definition of the propagation delays $\tau_{\ell, m}(t)$ given by (13), we find 


$$
\begin{aligned}
& R_{H}(t, f ; \Delta t, \Delta f)=\Pi_{T_{0}}(t) \Pi_{T_{0}}(t-\Delta t) \sum_{\ell=1}^{L} \sum_{p=1}^{L} \sum_{m=1}^{M} \sum_{q=1}^{M} E\left\{g_{\ell}^{T} g_{p}^{T}\right\} E\left\{g_{m}^{R} g_{q}^{R}\right\} E\left\{\exp \left\{j\left(\theta_{\ell}^{T}-\theta_{p}^{T}\right)\right\}\right\} E\left\{\exp \left\{j\left(\theta_{m}^{R}-\theta_{q}^{R}\right)\right\}\right\} \\
& \cdot E\left\{\exp \left\{j 2 \pi\left[\left(f_{c}+f\right) \tau_{\ell, m}(t-\Delta t)-\left(f_{c}+f+\Delta f\right) \tau_{p, q}(t)\right]\right\}\right\}, \\
& R_{H}(t, f ; \Delta t, \Delta f)=\Upsilon(t-\Delta t) \sum_{\ell=1}^{L} \sum_{m=1}^{M} E\left\{\left|g_{\ell}^{T}\right|^{2}\right\} E\left\{\left|g_{m}^{R}\right|^{2}\right\} E\left\{\exp \left\{j 2 \pi\left[\Delta t f_{\ell, m}^{D}\left(\frac{f_{c}+f}{f_{c}}\right)-\Delta f \tau_{\ell, m}(t)\right]\right\}\right\}
\end{aligned}
$$

The propagation delays $\tau_{\ell, m}(t)$ are statistically equivalent random processes for all $\ell \in\left\{1,2, \ldots, \operatorname{card}\left\{S_{T}\right\}\right\}$ and $m \in\left\{1,2, \ldots, \operatorname{card}\left\{S_{R}\right\}\right\}$. For this reason, we can take the third expectation out of the double summation in the latest equation. Then, taking account of (19), we get to the result presented in (26).

\section{B. Derivation of (45)}

Assuming that $G_{T}\left(\phi_{T}\right)=r_{T}, \forall \phi_{T}, G_{T}=r_{R}, \forall \phi_{R}$, and $\mathbf{D}=$ $[D, 0]^{\dagger}$, we can express (32) as

$$
D_{k}\left(\phi_{k}\right)=\frac{D}{2}+q_{k} r_{k} \cos \left(\phi_{k}\right), \quad k \in\{T, R\} .
$$

Substituting this result into (34), we find

$$
\begin{aligned}
& R_{H}^{k}(t, f ; \Delta t, \Delta f)=\zeta_{k} \Upsilon(t, \Delta t) \\
& \quad \cdot \int_{-\pi}^{\pi} \exp \left\{j 2 \pi\left[\Delta t f_{\max }^{k} \cos \left(\phi_{k}-\gamma_{k}\right)\left(\frac{f_{c}+f}{f_{c}}\right)-\Delta f\left(\frac{D / 2+r_{k}\left[1+q_{k} \cos \left(\phi_{k}\right)\right]}{C_{l}}-t \frac{f_{\max }^{k} \cos \left(\phi_{k}-\gamma_{k}\right)}{f_{c}}\right)\right]\right\} \\
& \quad \cdot p_{\phi}^{k}\left(\phi_{k}\right) d \phi_{k} .
\end{aligned}
$$

After some simple algebraic manipulations, we obtain

$$
\begin{aligned}
& R_{H}^{k}(t, f ; \Delta t, \Delta f)=\zeta_{k} \Upsilon(t, \Delta t) \\
& \cdot \exp \left\{\frac{-j \pi\left(D+2 r_{k}\right) \Delta f}{C_{l}}\right\} \\
& \cdot \int_{-\pi}^{\pi} \exp \left\{j 2 \pi\left[\cos \left(\phi_{k}\right) B_{c}^{k}+\sin \left(\phi_{k}\right) B_{s}^{k}\right]\right\} \\
& \cdot p_{\phi}^{k}\left(\phi_{k}\right) d \phi_{k},
\end{aligned}
$$

where $B_{c}^{k}$ and $B_{s}^{k}$ are defined in (46a) and (46b). Substituting the von Mises PDF into the above equation and making use of [38, Eq. (3.338-4)], we have

$$
\begin{aligned}
& R_{H}^{k}(t, f ; \Delta t, \Delta f) \\
& =\frac{\zeta_{k} \Upsilon(t, \Delta t) \exp \left\{-j \pi\left(D+2 r_{k}\right) \Delta f / C_{l}\right\}}{I_{0}\left(\kappa_{k}\right)} \\
& \quad \cdot I_{0}\left(\left\{\left[\kappa_{k} \cos \left(\mu_{k}\right)+j 2 \pi B_{c}^{k}\right]^{2}\right.\right. \\
& \left.\left.+\left[\kappa_{k} \sin \left(\mu_{k}\right)+j 2 \pi B_{s}^{k}\right]^{2}\right\}^{1 / 2}\right) .
\end{aligned}
$$

Finally, from (33), we obtain the expression presented in (45) for $R_{H}(t, f ; \Delta t, \Delta f)$.

\section{Disclosure}

The material in this paper is based on "Modeling of Nonstationary Double-Rayleigh Fading Channels for Mobile-toMobile Communications," by C. A. Gutiérrez, J. M. LunaRivera, and D. U. Campos-Delgado, which appeared in the proceedings of the 2016 European Wireless Conference (EW2016), Oulu, Finland, March 2016 (ㄷ 2016 IEEE).

\section{Conflicts of Interest}

The authors declare that there are no conflicts of interest regarding the publication of this paper.

\section{Acknowledgments}

The authors acknowledge the financial support provided by CONACYT for the development of this research work: Project Grants nos. 236188 and 241272.

\section{References}

[1] G. Araniti, C. Campolo, M. Condoluci, A. Iera, and A. Molinaro, "LTE for vehicular networking: a survey," IEEE Communications Magazine, vol. 51, no. 5, pp. 148-157, 2013.

[2] G. Karagiannis, O. Altintas, E. Ekici et al., "Vehicular networking: a survey and tutorial on requirements, architectures, 
challenges, standards and solutions," IEEE Communications Surveys and Tutorials, vol. 13, no. 4, pp. 584-616, 2011.

[3] J. B. Kenney, "Dedicated short-range communications (DSRC) standards in the United States," Proceedings of the IEEE, vol. 99, no. 7, pp. 1162-1182, 2011.

[4] P. Bello, "Characterization of randomly time-variant linear channels," IEEE Transactions on Communications, vol. 11, no. 4, pp. 360-393, 1963.

[5] H. Schulze and C. Lüders, Theory and Applications of OFDM and CDMA, John Wiley \& Sons, Ltd, Chichester, UK, 2005.

[6] A. Molisch, Wireless Communications, John Wiley and Sons, Chichester, UK, 2005.

[7] N. Costa and S. Haykin, Multiple-Input, Multiple-Output Channel Models: Theory and Practice, Wiley, New York, USA, 2010.

[8] C. F. Mecklenbraüker, A. F. Molisch, J. Karedal et al., "Vehicular channel characterization and its implications for wireless system design and performance," Proceedings of the IEEE, vol. 99, no. 7, pp. 1189-1212, 2011.

[9] R. H. Clarke, "A statistical theory of mobile-radio reception," The Bell System Technical Journal, vol. 47, no. 6, pp. 957-1000, 1968.

[10] O. Renaudin, V.-M. Kolmonen, P. Vainikainen, and C. Oestges, "Non-stationary narrowband MIMO inter-vehicle channel characterization in the 5-GHz band," IEEE Transactions on Vehicular Technology, vol. 59, no. 4, pp. 2007-2015, 2010.

[11] L. Bernadó, T. Zemen, F. Tufvesson, A. F. Molisch, and C. F. Mecklenbräuker, "The (in-) validity of the WSSUS assumption in vehicular radio channels," in Proceedings of the IEEE 23rd International Symposium on Personal, Indoor and Mobile Radio Communications (PIMRC '12), pp. 1757-1762, Sydney, NSW, Australia, September 2012.

[12] A. Roivainen, P. Jayasinghe, J. Meinilau, V. Hovinen, and M. Latva-Aho, "Vehicle-to-vehicle radio channel characterization in urban environment at $2.3 \mathrm{GHz}$ and $5.25 \mathrm{GHz}$," in Proceedings of the 2014 25th IEEE Annual International Symposium on Personal, Indoor, and Mobile Radio Communication, IEEE PIMRC 2014, pp. 63-67, Washington, DC, USA, September 2014.

[13] D. W. Matolak and J. Frolik, "Worse-than-rayleigh fading: experimental results and theoretical models," IEEE Communications Magazine, vol. 49, no. 4, pp. 140-146, 2011.

[14] L. Rubio, J. Reig, V. M. Rodrigo-Peñarrocha, H. Fernández, and S. Loredo, "Analysis of small-scale fading distributions in vehicular-to-vehicular communications," Mobile Information Systems, vol. 2016, Article ID 9584815, 7 pages, 2016.

[15] P. S. Bithas, K. Maliatsos, and A. G. Kanatas, "The Bivariate Double Rayleigh Distribution for Multichannel Time-Varying Systems," IEEE Wireless Communications Letters, vol. 5, no. 5, pp. 524-527, 2016.

[16] B. Talha and M. Pätzold, "A geometrical three-ring-based model for MIMO mobile-to-mobile fading channels in cooperative networks," Eurasip Journal on Advances in Signal Processing, vol. 2011, Article ID 892871, 2011.

[17] J. Karedal, F. Tufvesson, N. Czink et al., "A geometry-based stochastic MIMO model for vehicle-to-vehicle communications," IEEE Transactions on Wireless Communications, vol. 8, no. 7, pp. 3646-3657, 2009.

[18] M. Walter, D. Shutin, and U.-C. Fiebig, "Delay-dependent doppler probability density functions for vehicle-to-vehicle scatter channels," IEEE Transactions on Antennas and Propagation, vol. 62, no. 4, pp. 2238-2249, 2014.
[19] Y. Yuan, C.-X. Wang, Y. He, M. M. Alwakeel, and E.-H. M. Aggoune, "3D Wideband Non-Stationary Geometry-Based Stochastic Models for Non-Isotropic MIMO Vehicle-to-Vehicle Channels," IEEE Transactions on Wireless Communications, vol. 14, no. 12, pp. 6883-6895, 2015.

[20] C. A. Gutierrez, M. Patzold, W. Dahech, and N. Youssef, "A Non-WSSUS Mobile-to-Mobile Channel Model Assuming Velocity Variations of the Mobile Stations," in Proceedings of the 2017 IEEE Wireless Communications and Networking Conference (WCNC), pp. 1-6, San Francisco, CA, USA, March 2017.

[21] J. T. Gutiérrez-Mena, C. A. Gutiérrez, J. M. Luna-Rivera, D. U. Campos-Delgado, and J. V. Castillo, "Geometry-based statistical modeling of non-stationary MIMO vehicle-to-vehicle channels," in Proceedings of the 5th ACM Symposium on Development and Analysis of Intelligent Vehicular Networks and Applications, DIVANet 2015, pp. 85-92, Cancun, Mexico, November 2015.

[22] C. A. Gutiérrez, M. Luna-Rivera, and D. U. Campos-Delgado, "Modeling of non-stationary double-Rayleigh fading channels for mobile-to-mobile communications," in Proceedings of the 22nd European Wireless Conference, EW 2016, pp. 131-136, Oulu, Finland, May 2016.

[23] W. Dahech, M. Pätzold, C. A. Gutiérrez, and N. Youssef, "A Non-Stationary Mobile-to-Mobile Channel Model Allowing for Velocity and Trajectory Variations of the Mobile Stations," IEEE Transactions on Wireless Communications, vol. 16, no. 3, pp. 1987-2000, 2017.

[24] A. G. Zajić and G. L. Stüber, "Three-dimensional modeling and simulation of wideband MIMO mobile-to-mobile channels," IEEE Transactions on Wireless Communications, vol. 8, no. 3, pp. 1260-1275, 2009.

[25] J. Chen and T. G. Pratt, "A three-dimensional geometrybased statistical model of $2 \times 2$ dual-polarized MIMO mobileto-mobile wideband channels," Modelling and Simulation in Engineering, vol. 2012, Article ID 756508, 2012.

[26] X. Cheng, Q. Yao, M. Wen, C. Wang, L. Song, and B. Jiao, "Wideband channel modeling and intercarrier interference cancellation for vehicle-to-vehicle communication systems," IEEE Journal on Selected Areas in Communications, vol. 31, no. 9, pp. 434-448, 2013.

[27] D. K. Cheng, Field and Wave Electromagnetics, Addison-Wesley, New York, 2nd edition, 1989.

[28] M. Pätzold and B. Talha, "On the statistical properties of sumof-cisoids-based mobile radio channel simulators," in Proceedings of the Proc. 10th International Symposium on Wireless Personal Multimedia Communications (WPMC07, pp. 394-400, Jaipur, India, December 2007.

[29] B. O. Hogstad, C. A. Gutiérrez, M. Pätzold, and P. M. Crespo, "Classes of sum-of-cisoids processes and their statistics for the modeling and simulation of mobile fading channels," EURASIP Journal on Wireless Communications and Networking, vol. 2013, no. 1, pp. 1-15, 2013.

[30] J. J. Jaime-Rodriguez, C. A. Gutierrez, D. U. Campos-Delgado, and J. M. Luna-Rivera, "First-order statistics analysis of two new geometrical models for non-WSSUS mobile-to-mobile channels," in Proceedings of the 12th IEEE International Conference on Wireless and Mobile Computing, Networking and Communications, WiMob 2016, New York, NY, USA, October 2016.

[31] G. Matz, "On non-WSSUS wireless fading channels," IEEE Transactions on Wireless Communications, vol. 4, no. 5, pp. 2465-2478, 2005.

[32] A. G. Zajić, G. L. Stüber, T. G. Pratt, and S. T. Nguyen, "Wideband MIMO mobile-to-mobile channels: Geometry-based 
statistical modeling with experimental verification," IEEE Transactions on Vehicular Technology, vol. 58, no. 2, pp. 517-534, 2009.

[33] M. Pätzold, B. O. Hogstad, and N. Youssef, "Modeling, analysis, and simulation of MIMO mobile-to-mobile fading channels," IEEE Transactions on Wireless Communications, vol. 7, no. 2, pp. 510-520, 2008.

[34] A. Papoulis and S. Pillai, Probability, Random Variables, and Stochastic Processes, McGraw-Hill, New York, NY, USA, 4th edition, 2002.

[35] R. He, O. Renaudin, V.-M. Kolmonen et al., "Characterization of quasi-stationarity regions for vehicle-to-vehicle radio channels," IEEE Transactions on Antennas and Propagation, vol. 63, no. 5, pp. 2237-2251, 2015.

[36] R. von Mises, “Über die 'Ganzzahligkeit' der Atomgewichte und verwandte Fragen," Physikalische Zeitschrift, vol. 19, pp. 490500, 1918.

[37] R. D. Yates and D. J. Goodman, Probability and Stochastic Processes: A Friendly Introduction for Electrical and Computer Engineers, John Wiley and Sons, New Jersey, 2nd edition, 2005.

[38] I. S. Gradshteyn and I. M. Ryzhik, Tables of Integrals, Series and Products, Academic Press, New York, USA, 7th edition, 2007. 


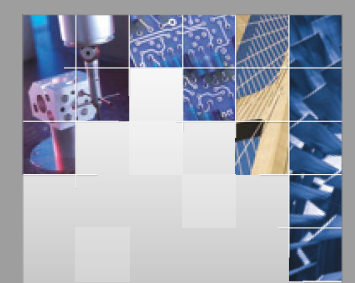

\section{Enfincering}
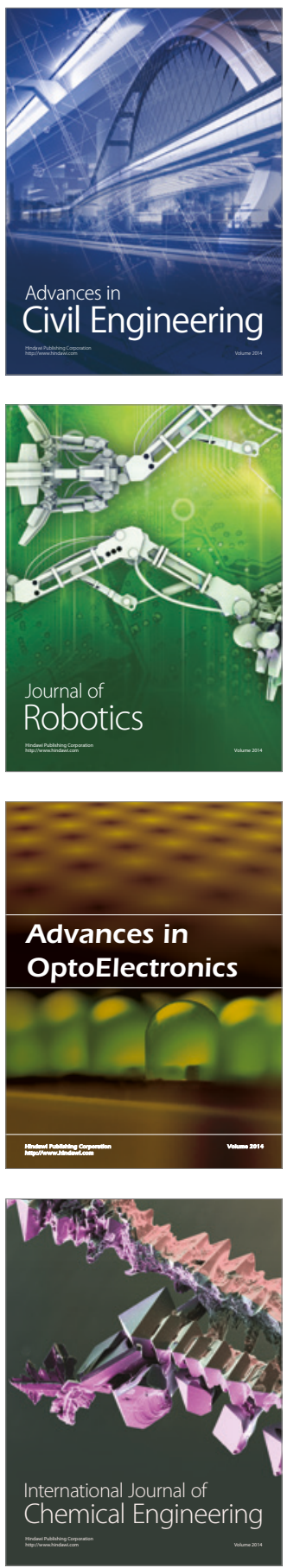

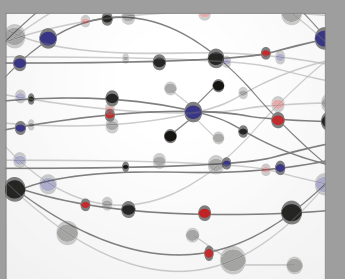

The Scientific World Journal

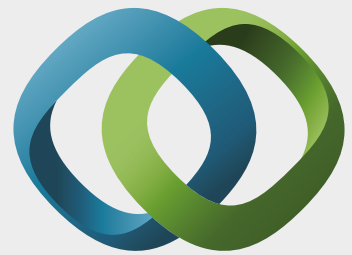

\section{Hindawi}

Submit your manuscripts at

https://www.hindawi.com
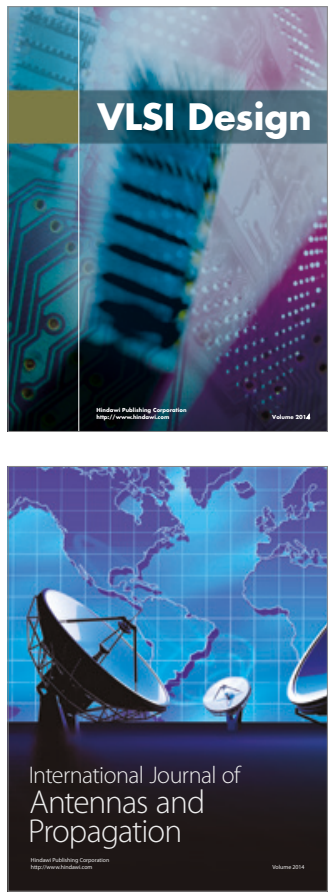

\section{Rotating}

Machinery
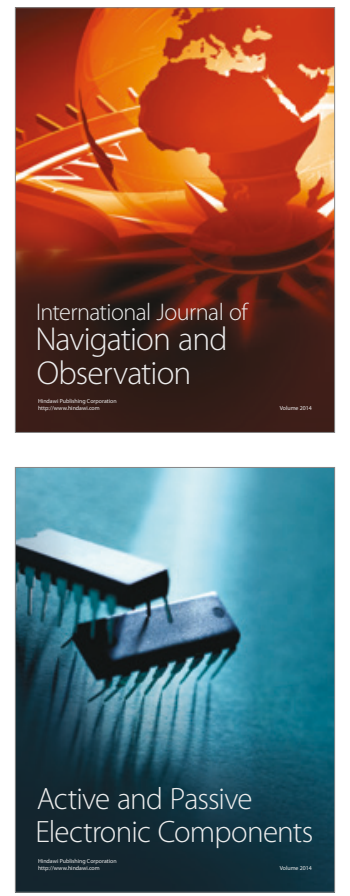
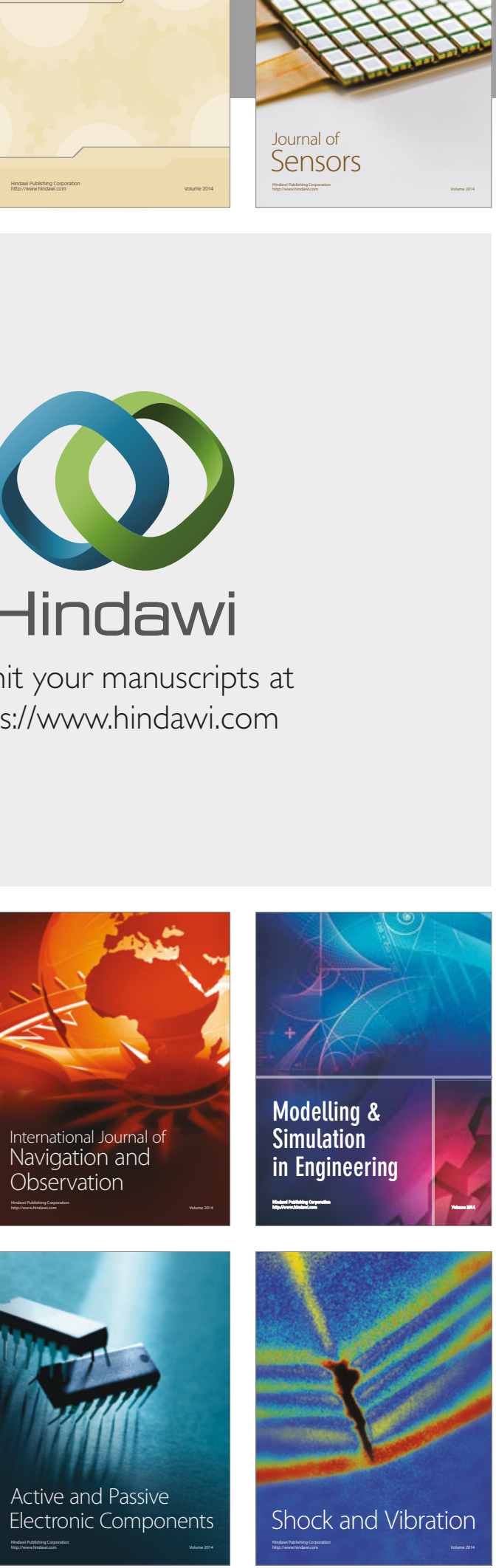
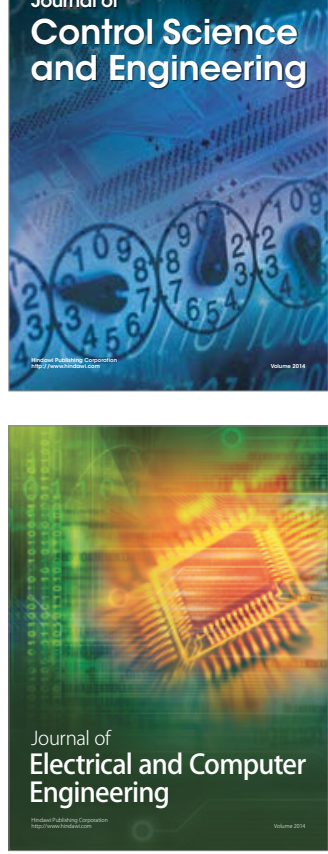

Distributed

Journal of

Control Science

and Engineering
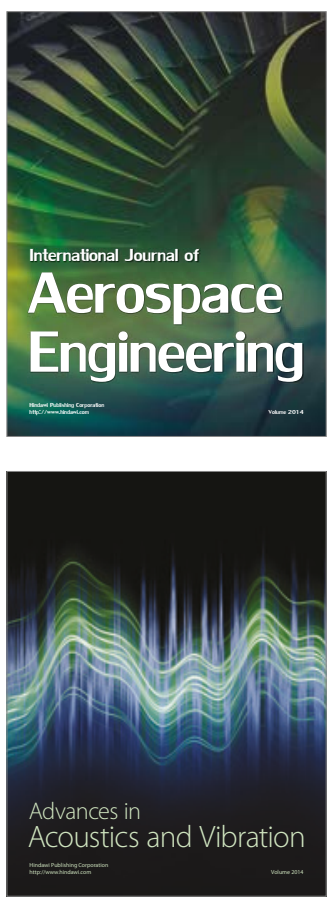

Sensor Networks 